\title{
Coagulation, fragmentation and radial motion of solid particles in protoplanetary disks ${ }^{\star}$
}

\author{
F. Brauer, C. P. Dullemond, and Th. Henning
}

\author{
Max-Planck-Institut für Astronomie, Königstuhl 17, 69117 Heidelberg, Germany \\ e-mail: brauer@mpia.de
}

Received 30 April 2007 / Accepted 11 November 2007

\begin{abstract}
The growth of solid particles towards meter sizes in protoplanetary disks has to circumvent at least two hurdles, namely the rapid loss of material due to radial drift and particle fragmentation due to destructive collisions. In this paper, we present the results of numerical simulations with more and more realistic physics involved. Step by step, we include various effects, such as particle growth, radial/vertical particle motion and dust particle fragmentation in our simulations. We demonstrate that the initial dust-to-gas ratio is essential for the particles to overcome the radial drift barrier. If this value is increased by a factor of 2 compared with the canonical value for the interstellar medium, km-sized bodies can form in the inner disk $(<2 \mathrm{AU})$ within $10^{4}$ yrs. However, we find that solid particles get destroyed through collisional fragmentation. Only with the unrealistically high-threshold velocities needed for fragmentation to occur $(>30 \mathrm{~m} / \mathrm{s})$, particles are able to grow to larger sizes in disks with low $\alpha$ values. We also find that less than $5 \%$ of the small dust grains remain in the disk after $1 \mathrm{Myr}$ due to radial drift, no matter whether fragmentation is included in the simulations or not. In this paper, we also present considerable improvements to existing algorithms for dust-particle coagulation, which speed up the coagulation scheme by a factor of $\sim 10^{4}$.
\end{abstract}

Key words. accretion, accretion disks - circumstellar matter - stars: formation - stars: pre-main-sequence - infrared: stars planetary systems: protoplanetary disks

\section{Introduction}

The coagulation of sub- $\mu \mathrm{m}$ dust particles is believed to be the initial step of planetesimal formation in disks around pre-mainsequence stars (Klahr \& Brandner 2006; Natta et al. 2007). Evidence for dust grain evolution beyond sizes that are found in the interstellar medium is provided by mid-infrared spectroscopy of disks around Herbig Ae/Be stars (Bouwman et al. 2001; van Boekel et al. 2003), T Tauri stars (Przygodda et al. 2003; Kessler-Silacci et al. 2007) and also around brown dwarfs (Apai et al. 2004, 2005, 2007; Sicilia-Aguilar et al. 2007). Millimeter interferometry indicates large populations of particles which have grown to even larger sizes, ranging up to several centimeters (Testi et al. 2003; Wilner et al. 2005; Rodmann et al. 2006).

All these observations give reason to model the evolution of particles in protostellar disks in order to explain the observational data (Dullemond \& Dominik 2004, 2005; Tanaka et al. 2005; Nomura \& Nakagawa 2006; D’Alessio et al. 2006; Ormel \& Cuzzi 2007). However, these theoretical investigations do not only attempt to model the evolution of the appearance of protostellar disks. They also unveil certain obstacles in the formation of planetesimals by particle coagulation (Youdin 2004; Dominik et al. 2007; Brauer et al. 2007).

One of these obstacles is the radial inward drift of solid bodies towards the central star as first dicussed by Whipple (1972) and Weidenschilling (1977a). The gas in a protostellar disk moves slightly sub-Keplerian due to a radial pressure gradient. For this reason, the dust which moves with near Keplerian

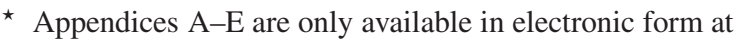
http://www . aanda.org
}

velocity feels a continuous head wind of gas. Hence, the particle loses angular momentum due to drag forces between gas and dust and spirals inward. If this radial drift of the particles is not prevented by some mechanism, then the solid particles drift into the inner evaporation zone and are lost for the process of planetesimal formation. To give an example, the radial drift time scale for meter-sized bodies at $1 \mathrm{AU}$ is $\sim 10^{2} \mathrm{yrs}$. Within this time scale these boulders at $1 \mathrm{AU}$ drift into the inner regions of the disk and evaporate. A possible way out of this problem is particle growth since the radial drift velocity is fairly dependent on the particle radius. For example, the drift velocity of meter-sized particles at $1 \mathrm{AU}$ in the disk is $\sim 50 \mathrm{~m} / \mathrm{s}$, but the radial drift velocity of $10 \mathrm{~m}$ sized bodies is already 10 times lower. Therefore, swift particle growth could prevent the particles from drifting into the evaporation zone. However, the general disk evolution comprises a considerable particle loss due to evaporation which is hard to prevent. This problem is a major topic of this paper. Other sublimation zones of the disk, e.g. the snow line at $\sim 2 \mathrm{AU}$ (Lecar et al. 2006), could also play a role for particle drift and coagulation processes. However, we will for now neglect this issue which will be the topic of a forthcoming paper.

Another obstacle is the fragmentation of solid particles. While low-velocity collisions lead to particle growth, high velocity impacts lead to destruction (Blum et al. 1998; Poppe et al. 1999; Blum \& Wurm 2000). For example, the relative particle velocity of meter-sized bodies in a protostellar disk can be more than $\sim 30 \mathrm{~m} / \mathrm{s}$ (Weidenschilling 1977a; Markiewicz et al. 1991). Benz (2000) found that meter-sized rocks appear unlikely to survive an impact with a relative low collision velocity of some $\mathrm{cm} / \mathrm{s}$. For porous objects, collision velocities higher than $4 \%$ of the sound speed lead to particle destruction (Sirono 2004). For 
this reason, the particle size of roughly a meter seems to pose an upper limit for particle coagulation.

These two obstacles, the radial drift barrier and the fragmentation barrier, are the issue of this paper. We present a disk model including the growth, the radial drift and the fragmentation of the particles. We show how these three effects change the evolution of the disk by including them step by step.

1. In the first step we only consider particle coagulation due to Brownian motion, vertical settling and turbulent mixing. This step shows to which sizes particles can grow if radial drift and fragmentation are neglected.

2. The second step includes the radial drift and the radial mixing of dust. The particles are now allowed to move inwards and to disappear into the evaporation zone. However, we investigate which disk parameters influence the drift time scales and for which parameters the dust particles overcome the drift barrier.

3. The last step also includes particle fragmentation. We show under which conditions, i.e. in which regions of the disk and for which disk parameters, it is possible for the dust to overcome this barrier.

The radial drift barrier is not only of interest for the radial drift itself. Particles close to this barrier are most susceptible to the motions of the gas and the gravitational effects of the dust. For example, particles can be trapped in very elongated gas vortices in magnetorotational turbulence (Balbus \& Hawley 1991; Barge $\&$ Sommeria 1995). These effects can slow down the radial drift by a factor of two (Johansen et al. 2006). Under certain conditions the solid particle layer itself may become gravitationally unstable (Johansen \& Youdin 2007). In high dust density regions, the particles contract due to their own gravity and may form a planetesimal within a few orbits (Johansen et al. 2007). Moreover, the flow of the gas and the dust can be unstable to the streaming instablity (Youdin \& Goodman 2005) which leads to particle clumping, and possibly also to a gravitational collapse of the dust. Apparently, the radial drift barrier is not only connected to the radial motion of the dust particles, but involves various other important effects as well. For this reason, it is vital to answer the question if particles can actually reach the size regime at which non-linear effects become of importance.

In this paper we will implement a $2+1$ dimensional model. The first dimension is the radial coordinate of the disk $r$, the second one is the height above the midplane $z$ and the third coordinate is the mass of the dust particles $m$. The dust may move radially due to radial drift and radial mixing. We will numerically solve the continuity equation for this problem for each particle species. The time evolution for the particle size distribution is determined by the coagulation equation. We will numerically solve this equation as well. In the vertical direction, we will always assume that each particle species is in vertical sedimentation/mixing equilibrium (Dubrulle et al. 1995; Cuzzi \& Weidenschilling 2006). Hence, we will not solve the time dependent continuity equation in the vertical direction as done for example by Dullemond \& Dominik (2005). Nor do we need to solve the coagulation equation at all $z$ explicitly. Instead, we solve the vertically integrated coagulation equation, which significantly saves computational time (cf. Appendix B). We also formulated the coagulation equation in an implicit way (cf. Appendix C) which saves another factor of $\sim 100$ of computer simulation time.

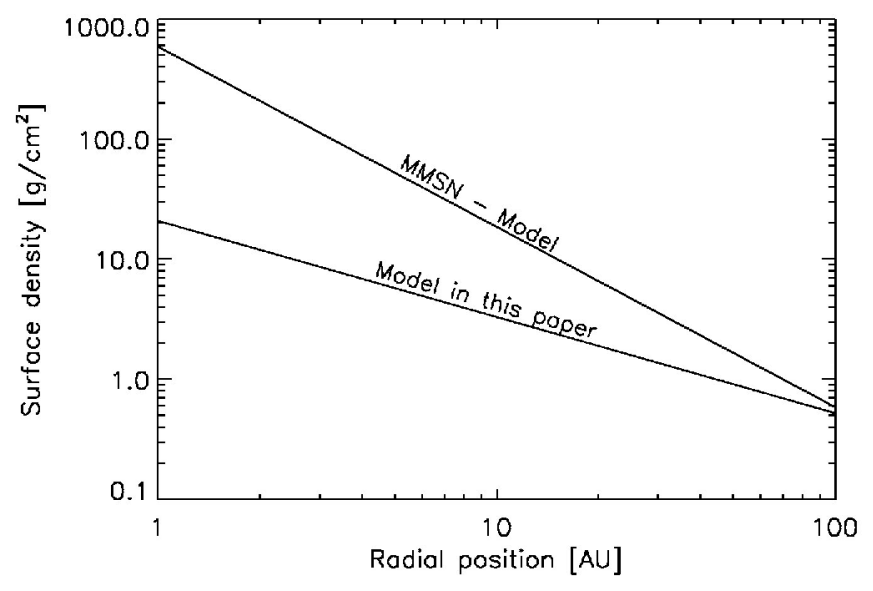

Fig. 1. The surface density distribution of the gas as a function of disk radius for the disk model discussed in the paper at hand and the MMSN model as discussed in Sect. 2. Note, that the surface densities at $1 \mathrm{AU}$ differ by more than one order of magnitude.

\section{Model equations}

\subsection{Disk model}

We consider a disk of mass $M_{\text {disk }}$ and an inner and an outer disk radius of $r_{\text {in }}=0.03 \mathrm{AU}$ and $r_{\text {out }}=150 \mathrm{AU}$. We adopt a central stellar mass of $0.5 M_{\odot}$ and a disk mass of $0.01 M_{\star}$ if not otherwise noted. The mass distribution of gas and dust inside the disk is given by the surface density $\Sigma$. This quantity generally depends on the distance to the central star $r$ and the azimuthal angle $\varphi$. However, since we assume a disk which is axisymmetric the surface density $\Sigma$ only depends on the radius $r$. We assume that this dependency can be described by a power law

$\Sigma=\Sigma_{0}\left(\frac{r}{1 \mathrm{AU}}\right)^{-\delta}$

The power law index of the surface density $\delta$ is set to be 0.8 in the course of this paper following Kitamura et al. (2002) and Andrews \& Williams (2007). The surface density at $1 \mathrm{AU}$, which is denoted by $\Sigma_{0}$, is chosen in a way that the condition

$2 \pi \int_{r_{\text {in }}}^{r_{\text {out }}} \Sigma(r) r \mathrm{~d} r=M_{\mathrm{disk}}$

holds. With the values mentioned above, the surface density of the gas at $1 \mathrm{AU}$ in the disk can be calculated to be $\sim 20 \mathrm{~g} / \mathrm{cm}^{2}$.

This surface density distribution of gas and dust, on which the dust particle coagulation calculations in this paper are based, differs significantly from the disk model which is usually referred to as the minimum mass solar nebula (MMSN) model (Weidenschilling 1977b; Hayashi 1981). The fundamental difference can be found in the distribution of the mass of gas and dust in the disk. In the MMSN disk model, the power law index of the surface density is $\delta=1.5$, while the disk model in this paper adopts $\delta=0.8$. Figure 1 shows the surface densities as a function of disk location for both disk models assuming a disk mass of $0.01 M_{\star}$. The MMSN model implies surface densities of $\sim 600 \mathrm{~g} / \mathrm{cm}^{2}$ at $1 \mathrm{AU}$ in the disk. With the disk model in this paper, we yields surface density values of $\sim 20 \mathrm{~g} / \mathrm{cm}^{2}$ which is more than one order of magnitude lower.

The actual distribution of mass in a protoplanetary nebula is still a matter of debate. There is evidence from meteoritics that the densities in the protosolar nebula in the planet forming region have been very high, implying disk masses much larger than the 
MMSN (Desch et al. 2002). On the other hand, resolved millimeter dust emission maps of protoplanetary nebula seem to indicate much lower surface densities (Andrews \& Williams 2007). However, millimeter dust observations of disks may not trace the radial profile of the gas mass density correctly since particle growth to larger sizes is expected to proceed more quickly in the inner parts of the disk than in the outer parts. For this reason, the dust continuum emission becomes flat even though the radial profile of the surface density might have a steep radial behaviour. Hence, analysing dust emission maps assuming a constant dust particle size throughout the disk likely leads to power law indices $\delta$ which are systematically shifted towards lower values.

The actual surface density distribution probably lies in between these two extreme cases, i.e. the MMSN model with $\delta=1.5$ and the observational median of $\delta=0.5$ (Andrews $\&$ Williams 2007). The surface density profile adopted in this paper is chosen to be between these two extremes. Our goal is to gain insight in dust disk evolution in a environment which might well be a likely scenario considering the $\delta$ range under discussion.

We assume that the gas in the disk is in a steady state, even for times as long as 1 Myr. Hence, the gas densities in our model do not change in time. We only focus on the dust component in the disk which evolves on a steady gas background. To unveil the robustness of this assumption, we compare the following time scales. Particle growth time scales are of the order of $\sim 10^{2}$ orbital times before fragmentation prevents further particle growth (cf. Sect. 3.3). Radial gas accretion velocities are of the order of $\sim 1 \ldots 10 \mathrm{~cm} / \mathrm{s}$ at 1 AU in the disk (Takeuchi \& Lin 2002). With regard to $1 \mathrm{AU}$, this leads to accretion time scales of the order of $10^{5} \mathrm{yrs}$, which is much larger than typical particle growth time scales. For example, Takeuchi \& Lin (2002) find that in the first $10^{4 \ldots 5} \mathrm{yrs}$, the gas surface density between 1 and 100 AU is hardly affected by viscous evolution. However, after $10^{5} \mathrm{yrs}$, the surface density of the gas may change significantly over time scales of several Myr (Reyes-Ruiz 2007). This introduces a systematic uncertainty in our dust evolution model regarding late evolutionary stages of $\mathrm{T}$ Tauri disks.

The temperature $T$ is assumed to be the midplane temperature of a disk irradiated under an angle of $\alpha_{\text {irr }}=0.05$ around a $\mathrm{T}$ Tauri star with a surface temperature of $T_{\star}=4000 \mathrm{~K}$ and a radius of $R_{\star}=2.5 R_{\odot}$. If we assume the disk to be isothermal in the vertical direction then the temperature is given by

$T=\alpha_{\text {irr }}^{1 / 4}\left(\frac{r}{R_{\star}}\right)^{-1 / 2} T_{\star}$.

With this dependency the temperature at $1 \mathrm{AU}$ is $204 \mathrm{~K}$. The evaporation temperature at $0.03 \mathrm{AU}$ is $\sim 1400 \mathrm{~K}$.

\subsection{Vertical structure of gas}

We consider a thin disk, which means that $z \ll r$. The quantity $z$ denotes the height above the midplane. Under this condition the vertical mass density distribution of the gas can be described by

$\rho_{\mathrm{g}}(z, r)=\frac{\Sigma(r)}{\sqrt{2 \pi} H} \exp \left(-z^{2} / 2 H^{2}\right)$.

In this expression the quantity $H$ denotes the pressure scale height of the gas given by $H=c_{\mathrm{s}} / \Omega_{\mathrm{k}}$, where $c_{\mathrm{s}}=\sqrt{k T / \mu}$ is the isothermal sound speed and $\Omega_{\mathrm{k}}=\sqrt{G M_{\star} / r^{3}}$ denotes the Kepler frequency. The quantities $k$ and $G$ are the Boltzman constant and the gravitational constant, respectively. The mean molecular weight $\mu$ is assumed to be $2.3 \mathrm{~m}_{\mathrm{p}}$ (mixture of molecular hydrogen and helium) where $m_{\mathrm{p}}$ is the mass of a proton.
With Eq. (4), the gas mass density in the midplane of the disk at 1 AU in our model with $\delta=0.8$ is given by $10^{-11} \mathrm{~g} / \mathrm{cm}^{3}$. Adopting the MMSN model with $\delta=1.5$ leads to a gas mass density of $4 \times 10^{-10} \mathrm{~g} / \mathrm{cm}^{3}$ which is more than one order of magnitude higher.

\subsection{Dust variables}

Before we introduce the vertical structure of the dust we define some variables that describe the dust distribution. We define $\rho_{\mathrm{d}}^{\text {tot }}$ to be the total mass of the dust per $\mathrm{cm}^{3}$ at a certain point in space. To describe the particle mass distribution we define a dust density $\rho_{\mathrm{d}}(m)$, normalised such that

$\rho_{\mathrm{d}}^{\mathrm{tot}}=\int_{0}^{\infty} \rho_{\mathrm{d}}(m) \mathrm{d} m$

Now, we introduce the number density $n$ by

$n(m)=\frac{\rho_{\mathrm{d}}(m)}{m}$.

This quantity gives the number of solid particles of a certain mass $m$ in a unity mass interval. Now, we can define the integrated number density $w$ and the surface density of the dust by

$\omega(m)=\int_{-\infty}^{+\infty} n(m) \mathrm{d} z$

and

$\Sigma_{\mathrm{d}}(m)=m \omega(m)$.

To implement these expressions in a computer program we have to introduce a mass grid $\left\{m_{\mathrm{k}}\right\}$ and a measure $\left\{\Delta m_{\mathrm{k}}\right\}$. With the definitions of the number density $N_{\mathrm{k}}$ and the dust density $\rho_{\mathrm{k}}$ on the mass grid

$N_{\mathrm{k}}=n\left(\bar{m}_{\mathrm{k}}\right) \Delta m_{\mathrm{k}} \quad$ and $\quad \rho_{\mathrm{k}}=\rho_{\mathrm{d}}\left(\bar{m}_{\mathrm{k}}\right) \Delta m_{\mathrm{k}}$

Eq. (6) implies

$\rho_{\mathrm{k}}=m_{\mathrm{k}} N_{\mathrm{k}}$.

The quantity $n\left(\bar{m}_{\mathrm{k}}\right)$ in Eq. (9) is an arbitrary value of the function $n$ within the interval $\Delta m_{\mathrm{k}}$ around $m_{\mathrm{k}}$. To define a surface density on a lattice we also have to introduce a vertical space grid $\left\{z_{1}\right\}$ and its measure $\left\{\Delta z_{1}\right\}$. The surface density is then given by

$\Sigma_{\mathrm{k}}=m_{\mathrm{k}} \sum_{l} N_{\mathrm{k}}\left(z_{\mathrm{l}}\right) \Delta z_{\mathrm{l}}$

\subsection{Vertical structure of the dust}

In a protostellar disk the scale height of the dust is determined by an equilibrium between two processes, namely the settling of the dust towards the midplane of the disk due to vertical gravity, and the vertical mixing of the dust due to turbulent diffusion. The more turbulence there is, the harder it is for the dust to form a thin layer since it is mixed up and transported back to the higher regions of the disk.

To describe turbulence, we will use the $\alpha$-prescription of Shakura \& Sunyaev (1973). In this prescription the turbulent diffusion coefficient of the gas at a certain radius $r$ in the disk is parameterized by the scale height of the gas $H$ and the isothermal sound speed $c_{\mathrm{s}}$ by

$D_{\mathrm{g}}=\alpha c_{\mathrm{s}} H$. 
The dimensionless parameter $\alpha$ determines the amount of turbulence in the disk. Observations suggest a turbulent $\alpha$ value of $10^{-3}$ (Hartmann et al. 1998). Numerical simulations of the magneto rotational instability (Balbus \& Hawley 1991) yield turbulence parameters of the same order of magnitude (Brandenburg et al. 1995; Hawley et al. 1995; Sano et al. 2004). Moreover, Weidenschilling (1980) showed that there is a minimal amount of turbulence in every protostellar disk corresponding to an $\alpha$ value of about $10^{-6}$.

In addition to the $\alpha$-value we have to introduce a second dimensionless number in order to describe the vertical structure of the dust. This so-called Stokes number is defined by

$\mathrm{St}_{\mathrm{k}}=\Omega_{\mathrm{k}} \frac{a_{\mathrm{k}} \rho_{\mathrm{s}}}{c_{\mathrm{s}} \rho_{\mathrm{g}}} \alpha^{2 q-1}$.

The variable $a_{\mathrm{k}}$ and $\rho_{\mathrm{s}}$ denote the radius of the dust particle of mass $\mathrm{k}$ and its material density, respectively. If the Stokes number is much smaller than unity, then the dust particles are strongly coupled to the gas. In this case, the motions of the dust are basically the motions of the gas and both components have the same behaviour with regard to diffusion. If St exceeds unity, then the particles decouple from the gas and are hardly influenced by the turbulent motions of the gas. The turbulence parameter $q$ in Eq. (13) determines whether turbulent diffusion in the disk is realized by big turbulent eddies moving slow $(q=1)$ or by small turbulent eddies moving fast $(q=0)$. Throughout this paper we will assume that $q=1 / 2$ following Cuzzi et al. (2001) and Schräpler \& Henning (2004) unless otherwise stated.

The dimensionless turbulence parameter $q$ is also connected with the velocity $v_{\mathrm{t}}$ of the large turbulent eddies,

$v_{\mathrm{t}}=\alpha^{q} c_{\mathrm{s}}$,

which significantly influences the relative turbulent velocities of the dust (cf. Sect. 2.6.1) and, hence, its coagulation and fragmentation time scales. Various authors have used quite different values for $q$ during the past decades which led to very different turbulent eddy velocities and, hence, different relative particle velocities produced by turbulence. While Morfill (1988), Weidenschilling (1988) or Weidenschilling \& Cuzzi (1993) use turbulent gas velocities of $\alpha c_{\mathrm{s}}$, which implies $q=1$, more recent publications explicitly derived $q=1 / 2$ which leads to $v_{\mathrm{t}}=\sqrt{\alpha} c_{\mathrm{s}}$ (Dubrulle et al. 1995; Cuzzi et al. 2001; Cuzzi \& Weidenschilling 2006). If $q$ exceeds $1 / 2$ then the time scale of the largest eddy becomes larger than an orbital time scale since $\tau_{\text {eddy }} \sim \alpha^{1-2 q} / \Omega_{\mathrm{k}}$. Turn over frequencies smaller than the Kepler frequency appear unphysical to us. Therefore, we follow Cuzzi et al. (2001) and adopt $q=1 / 2$.

With the two dimensionless numbers, $\alpha$ and $\mathrm{St}$, the scale height of the dust $h_{\mathrm{k}}$ of a certain grain mass $m_{\mathrm{k}}$ is given by (Dubrulle et al. 1995; Cuzzi \& Weidenschilling 2006; Brauer et al. 2007)

$\left(\frac{h_{\mathrm{k}}}{H}\right)^{2}=\frac{\alpha}{\min \left(\mathrm{St}_{\mathrm{k}}, 1 / 2\right)\left(1+\mathrm{St}_{\mathrm{k}}\right)}$.

Since the dust scale height $h_{\mathrm{k}}$ can not exceed the gas scale height $H$ we restrict $h_{\mathrm{k}}$ to be at most $H$. With this last expression the vertical structure of the dust particles with mass $m_{\mathrm{k}}$ is given by

$\rho_{\mathrm{k}}(r, z)=\frac{\Sigma_{\mathrm{k}}}{\sqrt{2 \pi} h_{\mathrm{k}}} \exp \left(-z^{2} / 2 h_{\mathrm{k}}^{2}\right)$.

In this equation $\Sigma_{\mathrm{k}}$ denotes the surface density of the dust with mass $m_{\mathrm{k}}$.

\subsection{Radial motion of the dust}

In this section we will present the equations of radial motion for solid particles. We first recapitulate the radial drift of individual particles and particle motion due to gas accretion. After this we introduce the equations for radial mixing due to turbulent diffusion. Finally, we will discuss the continuity equation.

The following equations, which describe the radial drift of individual dust particles, are valid as long as the dust-to-gas ratio $\epsilon$ does not exceed unity. If, however, the dust density becomes larger than the gas density then the dust starts to have a backreaction on the motion of the gas in a non negligible way. One possible scenario, where the dust-to-gas ratio can exceed unity, is when the particles settles into a thin midplane layer due to low turbulence in the disk. In this dense midplane layer these socalled collective effects can become of importance and the radial drift equations have to be modified in an appropriate way. This was discussed in detail by Nakagawa et al. (1986).

\subsubsection{Radial drift of individual particles}

We consider two different sources for the radial drift of solid particles. The first one is the radial drift of individual particles itself. The second source is due to the accretion process of the gas.

The dust particles behave entirely independently and the gas is assumed not to be affected by the dust at all. The crucial particle characteristics, that determine the drift of solid particles, is the Stokes number introduced in the last section. In other words, the coupling strength between the gas and the dust determines the radial drift. With this quantity the radial drift of individual dust particles of mass $m_{\mathrm{k}}$ is given by (Whipple 1972; Weidenschilling 1977a)

$v_{\text {dust, } \mathrm{k}}=-\frac{2 v_{n}}{\mathrm{St}_{\mathrm{k}}+\frac{1}{\mathrm{St}_{\mathrm{k}}}}$.

The radial drift of the dust is maximal for $\mathrm{St}_{\mathrm{k}}=1$. For this reason, the radial drift barrier can be regarded as a region around $\mathrm{St}_{\mathrm{k}}=1$. The maximum drift velocity $v_{n}$ in the last equation can be calculated according to

$v_{n}=\frac{c_{\mathrm{s}}^{2}}{2 V_{\mathrm{k}}}\left(\delta+\frac{7}{4}\right)$.

With the numbers mentioned in the last section the last expression yields a maximum drift velocity of $45 \mathrm{~m} / \mathrm{s}$ at 1 AU. Since the power law index of the temperature is $-1 / 2$ the maximum drift velocity $v_{\mathrm{N}}$ is independent of the location in the disk.

The second source for radial velocity of the dust is due to the accretion process of the gas. In a gaseous disk angular momentum is transported to the outer regions of the disk under the action of viscous stress. This transport of angular momentum is connected with a radial accretion velocity of the gas. This gas velocity also affects the motions of the dust. Small particles are strongly coupled to the motions of the gas and if the gas moves inwards then also the small dust particles move inwards. For larger particles, i.e. particles with Stokes number larger than unity, the motions of the gas become less and less important since larger dust particles decouple from the gas.

Takeuchi \& Lin (2002) have calculated the radial accretion velocity of the gas due to viscous stress,

$v_{\text {gas }}=-3 \alpha \frac{c_{\mathrm{s}}^{2}}{V_{\mathrm{k}}}\left(\frac{3}{2}-\delta\right)$. 


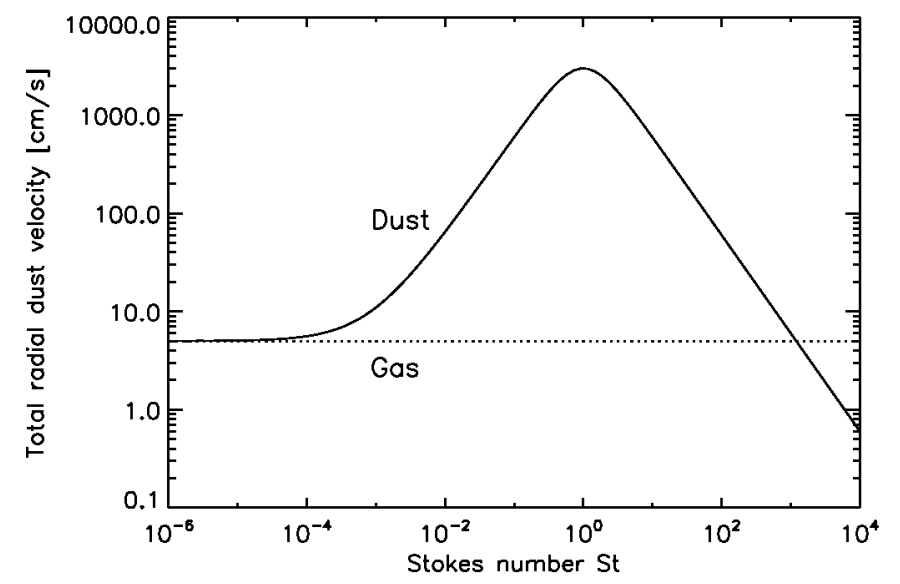

Fig. 2. The total inward radial velocity of a single dust particle as a function of Stokes number St (solid line) as discussed in Sect. 2.5.1. The dotted line denotes the accretion velocity of the gas. The turbulence parameter $\alpha$ is $10^{-3}$ in this calculation.

In the last expression we already adopted the temperature dependency $T \propto r^{-1 / 2}$ according to Eq. (3). The quantity $V_{\mathrm{k}}$ denotes the Kepler velocity $V_{\mathrm{k}}=\Omega_{\mathrm{k}} r$. With a turbulence parameter of $\alpha=10^{-3}$ and the values that were already mentioned in the last section the last equation yields a radial gas accretion velocity of $5 \mathrm{~cm} / \mathrm{s}$.

Now, the total radial drift velocity of solid particles of mass $m_{\mathrm{k}}$ is given by (Kornet et al. 2001)

$v_{\mathrm{dust}, \mathrm{k}}^{\mathrm{tot}}=v_{\mathrm{dust}, \mathrm{k}}+\frac{v_{\mathrm{gas}}}{1+\mathrm{St}_{\mathrm{k}}^{2}}$.

A plot of this total drift velocity is shown is Fig. 2.

\subsubsection{Radial mixing of the dust and the continuity equation}

Turbulence can be interpreted as a kind of diffusion. The turbulent diffusion coefficient for the gas $D_{\mathrm{g}}$ was already introduced in the last sections. The equivalent quantity for the dust of a certain grain mass is given by (Völk et al. 1980; Cuzzi et al. 1993; Schräpler \& Henning 2004)

$D_{\mathrm{d}, \mathrm{k}}=\frac{D_{\mathrm{g}}}{1+\mathrm{St}_{\mathrm{k}}}$.

This relation was already implicitly used in the expression for the dust layer thickness Eq. (15). For small particles, i.e. particles with $\mathrm{St}_{\mathrm{k}}<1$, the diffusion coefficient for the dust matches the diffusion coefficient for the gas. For Stokes numbers larger than unity $D_{\mathrm{d}, \mathrm{k}}$ decreases continuously since the particles more and more decouple from the turbulent gas. Recently, Youdin \& Lithwick (2007) have shown analytically that the diffusion coefficient of the dust rather decreases with $\left(1+\mathrm{St}^{2}\right)^{-1}$ than with $(1+\mathrm{St})^{-1}$. However, since we restricted the settling time scale to be $1 / \Omega_{\mathrm{k}}$ at most, our dust scale height formula equals the expression found by Youdin \& Lithwick (2007).

The continuity equation of the dust of a certain grain size including radial drift and turbulent mixing reads

$\dot{\Sigma}_{\mathrm{k}}+\frac{1}{r} \partial_{r}\left(r F_{\mathrm{k}}\right)=0$.

The dust mass flux $F_{\mathrm{k}}$ is given by

$F_{\mathrm{k}}=\Sigma_{\mathrm{k}} v_{\mathrm{dust}, \mathrm{k}}^{\text {tot }}-D_{\mathrm{d}, \mathrm{k}} \Sigma_{\mathrm{g}} \partial_{r}\left(\frac{\Sigma_{\mathrm{k}}}{\Sigma_{\mathrm{g}}}\right)$.
The first term on the right side is the mass flux for the radial drift of individual particles discussed in the last section. The second term is the mass flux due to turbulent diffusion (radial mixing).

\subsection{Dust coagulation}

Two particles of mass $m_{i}$ and $m_{j}$ in a protostellar disk tend to have different particle velocities $v_{i}$ and $v_{j}$ (Beckwith et al. 2000). For example, micrometer-sized particles are carried away with the gas while larger boulders, like meter-sized bodies, which are decoupled from the gas, are hardly affected by any gas motion. Small particles have high relative velocities due to Brownian motion even for equal-sized pairs. Larger particles have not. These relative velocities $\Delta v_{i j}$ lead to occasional collisions. The number of collisions per second between two particle species with number densities $N_{i}$ and $N_{j}$ can be calculated to be

$\frac{\text { collisions }}{\mathrm{s}}=\Delta v_{i j} \sigma_{i j} N_{i} N_{j}$

We make the simplification that we take for $\Delta v_{i j}$ the average relative velocity between the two dust particles. In principal, the relative velocities have stochastic variations, but we ignore them here. We assume that collisions lead to coagulation with a certain sticking probability $p_{\mathrm{c}}$. In general this probability depends on various particle parameters like the size of the particle, solid particle density, the "fluffiness" of the particles (Blum \& Wurm 2000). It also depends on the relative particle velocity $\Delta v_{i j}$. Small particles tend to stick to each other up to high relative velocities (Dominik \& Tielens 1997) while larger bodies show the tendency to fragment even for small relative velocities (Benz 2000). This sticking probability will be discussed in more detail in Sect. 2.8.

With the collision rate Eq. (24) we can calculate the number of dust particles per second with mass $m_{i}$ which coagulate with any dust particle of any mass,

$\dot{N}_{i}^{\text {Loss }}=\sum_{j} \Delta v_{i j} \sigma_{i j} p_{\mathrm{c}} N_{i} N_{j}$,

which corresponds to the loss term for the number density of particles with mass $m_{i}$. The factor $\Delta v_{i j} \sigma_{i j} p_{\mathrm{c}}$ is often called the coagulation kernel. The gain term for particles with mass $m_{i}$ due to the coagulation of smaller particles with mass $m_{\mathrm{k}}$ and $m_{j}$ reads

$\dot{N}_{i}^{\text {Gain }}=\sum_{m_{i}=m_{\mathrm{k}}+m_{j}} \Delta v_{\mathrm{k} j} \sigma_{\mathrm{k} j} p_{\mathrm{c}} N_{\mathrm{k}} N_{j}$.

Now we can introduce the full coagulation equation which is given by (Smoluchowski 1916)

$\dot{N}_{i}=\dot{N}_{i}^{\text {Gain }}-\dot{N}_{i}^{\text {Loss }}$.

The continuous formulation of the coagulation equation, as discussed by Safronov (1969), corresponds to a non-linear integrodifferential equation. However, this equation is rather difficult to solve both in its discrete or continuous version. This is only within the realms of possibility for very simple (and unfortunately unphysical) kernels. Therefore, we will solve the coagulation equation numerically. The algorithm we will make use of is described in detail in Appendix A.

\subsubsection{Relative dust particle velocities}

We will consider four different sources for relative particle velocities which lead to coagulation: Brownian motion, differential settling, turbulence and radial drift. 
First, let us focus on Brownian motion. Two particles of mass $m_{1}$ and $m_{2}$ in a region of the disk with temperature $T$ have an average statistical relative velocity due to Brownian motion given by

$\Delta v_{\mathrm{B}}=\sqrt{\frac{8 k T\left(m_{1}+m_{2}\right)}{\pi m_{1} m_{2}}}$.

This expression shows that relative thermal velocities are higher for smaller dust particles than for larger dust particles. Hence, the growth process due to Brownian motion is more effective for small particles than for large particles. For example, if we assume a temperature of $200 \mathrm{~K}$, a solid particle density of $1 \mathrm{~g} / \mathrm{cm}^{3}$ and micrometer-sized particles then the relative particle velocity due to Brownian motion is $0.2 \mathrm{~cm} / \mathrm{s}$. Particles of centimeter in size lead to a relative velocity of $10^{-7} \mathrm{~cm} / \mathrm{s}$. This particular example shows that there is practically no coagulation due to Brownian motion for particles much larger than micrometer size. In general, growth by Brownian motion leads to fractal structures and "fluffy" aggregates (cluster-cluster aggregates) (Ossenkopf 1993; Kempf et al. 1999). However, we will ignore these intrinsic properties of the dust particles in the course of this paper and assume a constant solid material density. See, however, Schmitt et al. (1997) or Ormel et al. (2007) for dust particle coagulation models including porosity at a fixed radius in the disk.

Differential settling is the second process that leads to relative velocities. If we assume that the solid particles are smaller than the mean free path of the gas then the equilibrium settling velocity is given by $z \mathrm{St} \Omega_{\mathrm{k}}$ (Dullemond \& Dominik 2004). In this expression St is the Stokes number introduced in Sect. 2.4. However, for Stokes numbers larger than unity, the equilibrium settling velocity model loses validity. Very large bodies $(\mathrm{St} \rightarrow \infty)$ above or below the midplane follow an orbit that is tilted with respect to the midplane. The settling velocity towards the midplane can not exceed the vertically projected Kepler velocity $z V_{\mathrm{k}} / r$ corresponding to this inclined orbit. For this reason we restrict the settling velocity to be the projected Kepler velocity at most. Considering this, we adopt the following settling velocity in our model,

$v_{\mathrm{S}}=\frac{z \mathrm{St} \Omega_{\mathrm{k}}}{1+\mathrm{St}}$.

The relative settling velocity between two particles of mass $m_{i}$ and $m_{j}$ at a height $z$ above the midplane then reads

$\Delta v_{\mathrm{S}}=z \Omega_{\mathrm{k}}\left|\frac{\mathrm{St}_{i}}{1+\mathrm{St}_{i}}-\frac{\mathrm{St}_{j}}{1+\mathrm{St}_{j}}\right|$.

The third source for relative velocities of particles in the disk is the radial drift which was discussed in detail in Sect. 2.5. The relative velocity in this case is simply the difference in the drift velocities

$\Delta v_{\mathrm{D}}=\left|v_{\text {dust }, \mathrm{i}}^{\text {tot }}-v_{\text {dust } \mathrm{j} j}^{\text {tot }}\right|$.

The fourth relative velocity between the particles is due to turbulence in the disk. Relative particle velocities produced by turbulence were calculated numerically by Völk et al. (1980) and Mizuno et al. (1988). Weidenschilling (1984) fitted these results with analytical formulas. Current work by Ormel \& Cuzzi (2007) shows that these expressions underestimate the turbulent relative velocities for particles with large Stokes numbers. In this paper, we will use the expressions calculated by Ormel \& Cuzzi (2007).

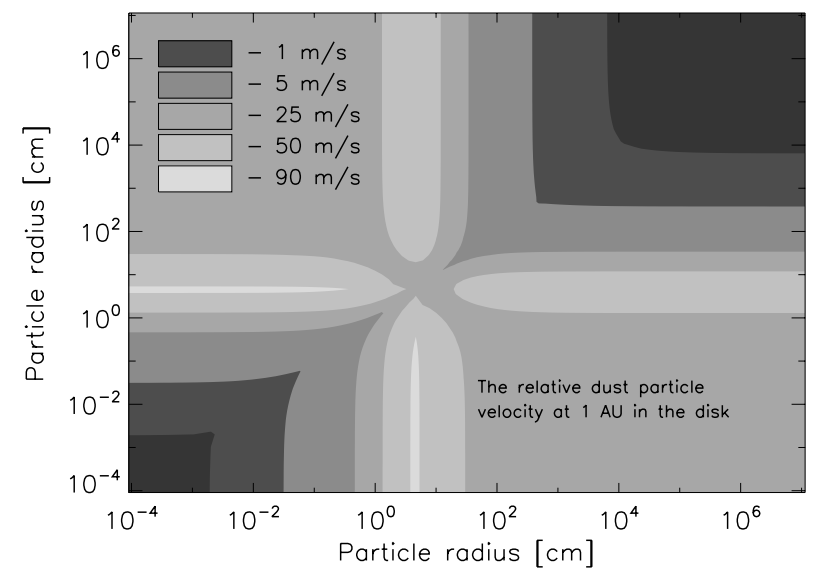

Fig. 3. Relative velocities of dust particles at $1 \mathrm{AU}$ in the disk as discussed in Sect. 2.6.1. This calculation includes Brownian motion, differential settling and relative turbulent velocities. In this calculation we adopted a turbulent $\alpha$ value of $10^{-3}$.

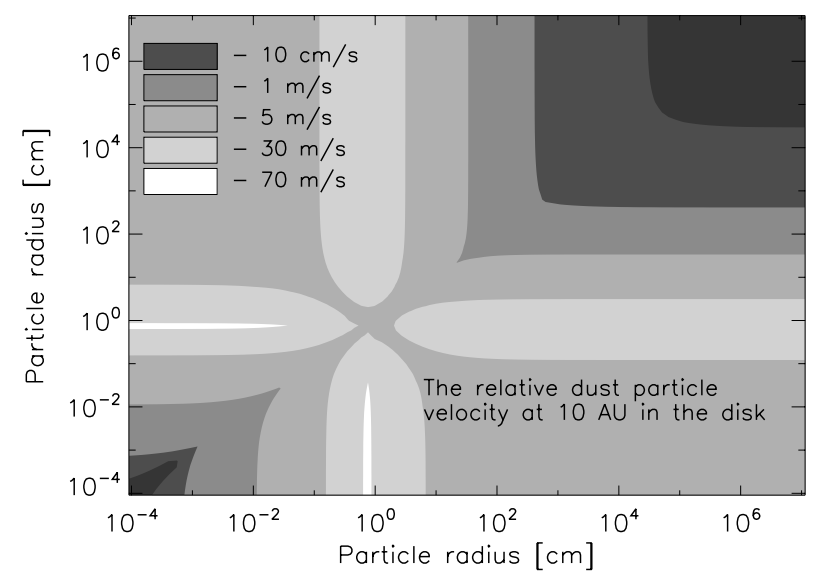

Fig. 4. As Fig. 3, but now at $10 \mathrm{AU}$ in the disk.

To give an impression of relative dust particle velocities in a protostellar disk, Fig. 3 shows a contour plot of this quantity at 1 AU including Brownian motion, differential settling, relative turbulent velocities and relative particle drift velocities. The same calculation at $10 \mathrm{AU}$ is shown in Fig. 4.

\subsection{Particle fragmentation}

Collisions between particle aggregates do not necessarily lead to particle growth. For sufficiently high relative collision velocities, the aggregates may fragment into smaller bodies. The critical threshold velocity for this destructive process generally depends on the mass of the colliding particles. More precisely, fragmentation tends to play a non-negligible role if the kinetic collisional energy of the particles is of the order of the internal binding energy of the particles (Borkowski \& Dwek 1995). Fragmentation velocities of aggregates are usually of the order of a few $\mathrm{cm} / \mathrm{s}$ up to several $10 \mathrm{~m} / \mathrm{s}$. While smaller particles tend to stick to each other up to high relative particle velocities (Dominik \& Tielens 1997) larger bodies show the tendency to fragment even for small relative velocities (Benz 2000). For simplicity, we will assume a fixed threshold velocity for particle destruction $v_{\mathrm{f}}$ which does not depend on the mass of the particles. However, we will investigate how the results of the simulations change if $v_{\mathrm{f}}$ is varied over a wide parameter range. The dependency of $v_{\mathrm{f}}$ on the 
particle mass will be investigated in the near future including laboratory results of dust particle collisions.

The result of destructive collisions between solid particles, i.e. the exact particle distribution after fragmentation, is still a matter of debate. Usually this particle distribution is described by a power-law,

$n(m) \mathrm{d} m \propto m^{-\xi} \mathrm{d} m$.

In this expression $n(m) \mathrm{d} m$ is the number of particles per unit volume within the mass range $[m, m+\mathrm{d} m]$. The last decades involved various attempts to determine the fragmentation parameter $\xi$. Mathis et al. (1977) and also Draine \& Lee (1984) showed that the extinction and scattering of starlight by interstellar dust can be reproduced by a power-law dependency following $\xi=1.83$. Experimental studies found values for $\xi$ ranging between 1.3 (low-velocity impacts) and 2 (catastrophic impacts) (Davis \& Ryan 1990; Blum \& Muench 1993). Steady state solutions between coagulation and fragmentation lead to $\xi=1.83$ as shown by Dohnanyi (1969). More recently, Tanaka et al. (1996) argued that the very general result $\xi=1.83$ is a direct implication of the self-similarity of the particle size distribution. In this paper we will assume the $\xi$-value 1.83 if not otherwise noted.

The process of fragmentation between particles which have the same mass is different from the fragmentation of particles whose mass differ by orders of magnitude. Two bodies of equal mass may destroy each other. Small dust grains, however, are not able to destruct a meter-sized body. But they can excavate a small crater in the larger target. This process is usually called "cratering". We will assume that cratering sets in if the mass of the colliding bodies differs by more than one order of magnitude. In this case, the smaller dust particle $m_{\mathrm{s}}$ excavates a crater which contains a factor $\chi$ times its own mass, i.e. $m_{\text {crater }}=\chi m_{\mathrm{s}}$. The parameter $\chi$ is set to unity if not otherwise noted. The mass of the smaller body and the crater ejecta are then redistributed according to Eq. (32). On the other hand, if the mass of the colliding particles differs by less than an order of magnitude, i.e. in the non-cratering case, then the total mass is redistributed following Eq. (32).

To illustrate the results of fragmentation Fig. 5 shows the outcome of a destructive collision as modeled in the paper at hand. The solid line shows the outcome of fragmentation in the case of cratering. The dotted line corresponds to the fragmentation results of two particles with the same mass.

\subsection{Coagulation and fragmentation probabilities}

If the collision velocity of two boulders is sufficiently large then the particles tend to fragment into smaller bodies instead of coagulating to larger aggregates. We will assume that the probability for fragmentation $p_{\mathrm{f}}$ only depends on the relative particle velocity $\Delta v$ and adopt the following expression for this probability,

$p_{\mathrm{f}}(\Delta v)=\left(\frac{\Delta v}{v_{\mathrm{f}}}\right)^{\psi} \Theta\left(v_{\mathrm{f}}-v\right)+\Theta\left(v-v_{\mathrm{f}}\right)$.

The two Heaviside step functions $\Theta$ ensure that the particles fragment with $100 \%$ probability if the relative particle velocity $\Delta v$ is larger than the critical fragmentation velocity $v_{\mathrm{f}}$. For $\Delta v<v_{\mathrm{f}}$ we assume that there is always a possibility for fragmentation given by $\left(v / v_{\mathrm{f}}\right)^{\psi}$. We will investigate the influence of the critical fragmentation velocity $v_{\mathrm{f}}$ and the index $\psi$. The value of $\psi$ is set to unity if not otherwise noted. The probability for

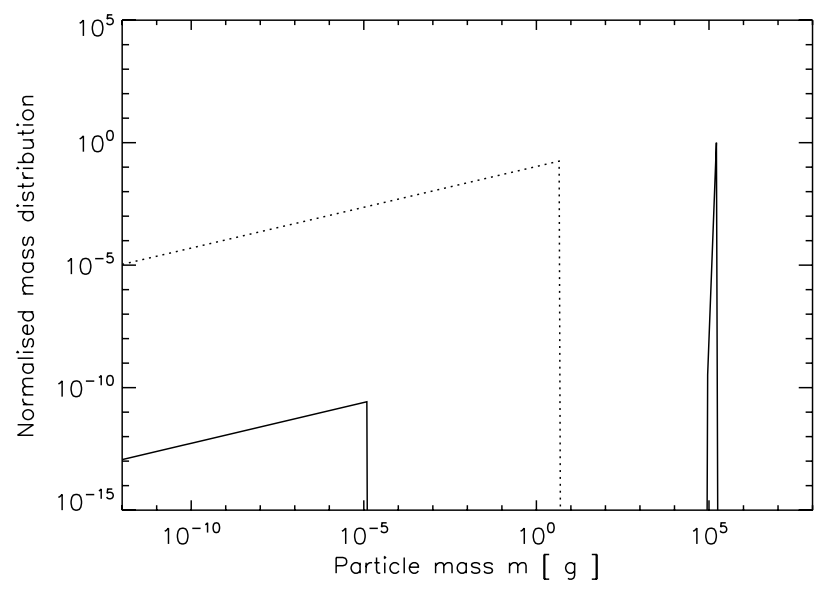

Fig. 5. The assumed fragmentation results of collisional destruction as discussed in Sect. 2.7. The solid line shows an example for cratering. The larger body has a mass of $\sim 10^{5} \mathrm{~g}$. The smaller dust grain, which is destroyed in this process, had a mass of $\sim 10^{-5} \mathrm{~g}$. The dotted line corresponds to the collision of two particles with the same mass. In this calculation the fragmentation parameter $\xi$ is assumed to be 1.83 which means that most of the fragmentation results are at the large end of the particle size distribution.

coagulation $p_{\mathrm{c}}$ is given by $p_{\mathrm{c}}=1-p_{\mathrm{f}}$. The last expression implies that the particles either coagulate or they fragment. We do not allow the particles to collide and not to undergo either the process of coagulation or fragmentation. However, just for the moment let us assume that $p_{\mathrm{f}}+p_{\mathrm{c}}<1$. If this last expression holds then the time scales for coagulation and fragmentation increase. However, this issue might be considered in a forthcoming paper.

\section{Simulation results}

In the following we will present the results of our numerical simulations. These simulations include various effects, for example, different particle growth mechanisms (Brownian motion, differential settling, etc.), the radial drift of the dust and particle fragmentation. To illustrate the influence of these effects on the particle growth, we will proceed in certain steps. In every step more and more effects will be included. In the first step we will consider the growth of the dust particles at various radii in the disk. In this part we do not allow the particles to move radially. In the second step, however, we will also include the radial motion of the dust which was discussed in detail in Sect. 2.5. In the last step we will additionally consider fragmentation.

\subsection{Step 1 - Coagulation only}

What are the growth time scales of the solid particles at different radii in the disk? To answer this question, we will not allow any radial motion of the particles. We glue the dust to a certain radial position even though the radial drift of the dust is potentially very high. We also do not allow particle fragmentation. The coagulation of solid particles at a fixed radius in the disk was for example also treated by Schmitt et al. (1997) and Dullemond \& Dominik (2005), Nakagawa et al. (1981), Tanaka et al. (2005) and recently Ciesla (2007). We assume that the mass of the disk is $1 \%$ of the central mass, an initial dust-to-gas ratio of $\epsilon_{0}=10^{-2}$ and a solid material density ${ }^{1}$ of $\rho_{\mathrm{s}}=1.6 \mathrm{~g} / \mathrm{cm}^{3}$. The

$110 \%$ silicate, $30 \%$ carbonaceous material and $60 \%$ ice. 


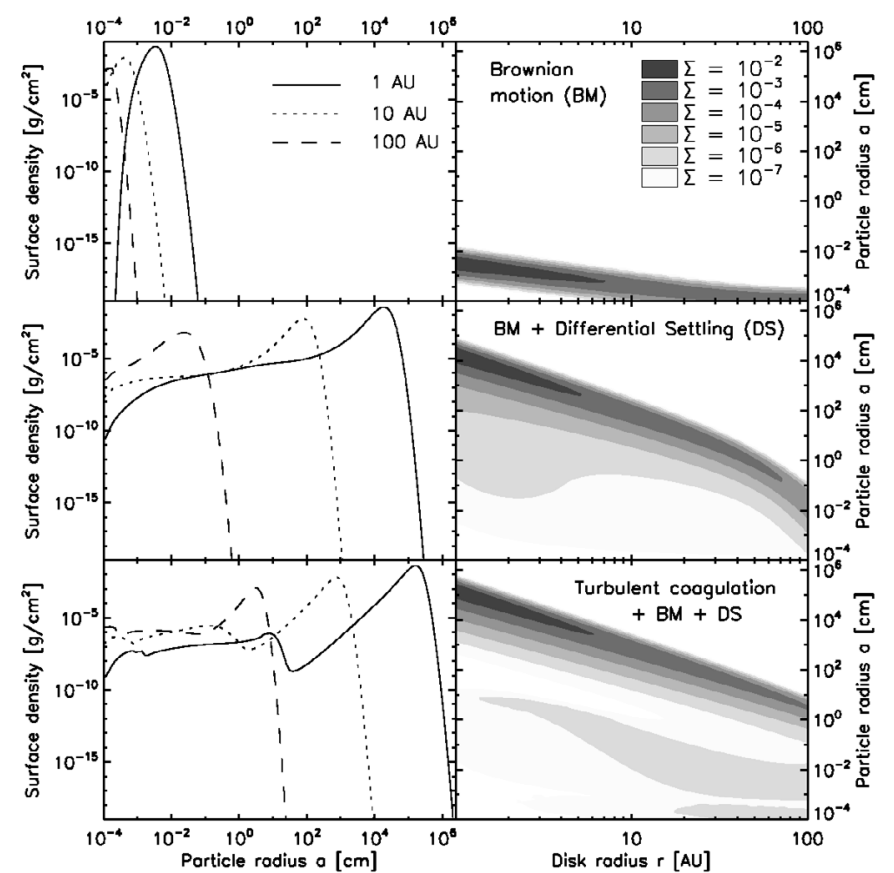

Fig. 6. These plots show the particle size distribution at different radii in the disk after 1 Myr of disk evolution as discussed in Sect. 3.1. The left and the right plot always belong together. From top to bottom more and more growth mechanisms are included in the simulations. The upper panel shows coagulation only due to Brownian motion (BM). The second panel shows BM and differential settling (DS). Finally, BM + DS and turbulent coagulation (TC) are shown in the lowest panel. The left plots show the surface density of the dust at 1, 10 and $100 \mathrm{AU}$ as a function of particle radius. On the right side the corresponding contour plots of the dust surface density are shown as a function of the radial location in the disk and the particle radius. In these simulations the radial drift as well as the fragmentation of the dust particles were neglected.

turbulent $\alpha$ parameter is $10^{-3}$ and the turbulent $q$ parameter is set to be $1 / 2$. At the beginning of each simulation the dust is equally distributed between a dust particle size of $0.5 \mu \mathrm{m}$ and $0.8 \mu \mathrm{m}$.

Let us first focus on the particle growth due to Brownian motion at different radii in the disk. The result of this simulation, i.e. the particle size distribution after $1 \mathrm{Myr}$, is shown in the upper panel of Fig. 6. According to these results dust particles grow from sub-micrometer to $\sim 30 \mu \mathrm{m}$ in radius in $1 \mathrm{Myr}$ at $1 \mathrm{AU}$ in the disk. At $10 \mathrm{AU}$ the particle distribution has a maximum for $a \sim 4 \mu \mathrm{m}$. At $100 \mathrm{AU}$ most of the dust is roughly a micrometer in size. We conclude that particle growth due to Brownian motion is not very effective, which is a well known result (Ossenkopf 1993; Schmitt et al. 1997; Dullemond \& Dominik 2005). However, Brownian motion is an important effect for the following reason. We calculate the relative velocities due to Browian motion, differential settling and turbulence for $a=0.6 \mu \mathrm{m}$ equal-sized particles at $1 \mathrm{AU}$ in the disk. While the relative particle velocity due to Brownian motion is $0.4 \mathrm{~cm} / \mathrm{s}$ the relative turbulent velocity is in the order of $10^{-8} \mathrm{~cm} / \mathrm{s}$. The relative velocity due to differential settling is practically zero. Dust particle growth due to differential settling or turbulence gets of importance only for larger particles. Therefore, Brownian motion is a trigger mechanism for the entire coagulation process which was noted before by Weidenschilling (1984).

Now, we will additionally include coagulation due to differential settling into our model. The result of this simulation is shown in the second panel of Fig. 6. This plot shows that particles have grown to more than $10^{4} \mathrm{~cm}$ in radius at $1 \mathrm{AU}$ in the disk after 1 Myr. This particle size is more than 6 orders of magnitude larger than the grain size after 1 Myr caused by Brownian motion. Most particles at $10 \mathrm{AU}$ and $100 \mathrm{AU}$ have grown to sizes of about $1 \mathrm{~m}$ and $100 \mu \mathrm{m}$, respectively. We conclude that differential settling is an effective growth mechanism which can create large boulders in the inner parts of the disk. Note that in our model the vertical mixing continuously allows the grains to go back up again and grow again by differential settling. Therefore, the maximal size formula of Safronov (1969) does not apply here.

Apart from the fact that particles grow to much larger sizes if differential settling is included, Fig. 6 also shows that there is always a certain amount of small particles that remains in the disk and that does not coagulate for at least 1 Myr. After this time, roughly $6 \%$ of the dust between 1 and $75 \mathrm{AU}$ is still present in grains $<1 \mathrm{~mm}$. The reason for this is the following. Not all of the dust particles coagulate at the same time. While a certain fraction of the dust has already grown to larger sizes and formed a thinner dust layer according to Eq. (15), a certain fraction of small dust remains in the higher regions above the midplane. These small dust particles high above the midplane are subject to a rather slow coagulation process. The dust densities above the midplane are low after most of the dust already settled closer to the midplane. This leads to long growth time scales according to Eq. (24). Larger particles close to the midplane can not sweep up the smaller particles above the midplane since turbulence is not able to stir them up so far. For this reason small particles remain in the disk for a long time.

We will now also include relative velocities of the particles caused by random turbulent motions. The result of this simulation is shown in Fig. 6 in the lower panel. This plot indicates that the dominant grain size at $1 \mathrm{AU}$, i.e. the grain size corresponding to the surface density maximum, changes by a factor of $\sim 10$ if turbulent coagulation is included in the simulation. The dominant particle radius at $1 \mathrm{AU}$ is $\sim 10^{5} \mathrm{~cm}$. At $100 \mathrm{AU}$, random turbulent motions also speed up the coagulation process which leads to particles of a few centimeters in radius after $1 \mathrm{Myr}$ of disk evolution. Without relative turbulent velocities included in the simulation, the particle radius was two orders of magnitude smaller.

\subsection{Step 2 - Coagulation and radial motion}

We will now include radial motion, both as transport and as extra source of relative velocities for coagulation. This significantly changes the results of the last section. We find that the radial drift of solid particles is so high that the dust drift into the evaporation zone long before larger particles in the disk can possibly form. This happens even though an additional source for coagulation is introduced which decreases the coagulation time scales. We will investigate if particles can in some way "break through" the radial drift barrier.

\subsubsection{Time evolution of the disk}

Figure 7 shows the time evolution of the model. This plot indicates that $\mathrm{cm}$-dm-sized particles form in the inner regions of the disk $(<2 \mathrm{AU})$ within the first $10^{3}$ yrs. Compared to the outer parts of the disk the formation of these particles appears rather quickly due to comparatively high gas and dust densities and high temperatures. With increasing distance from the central star the formation of larger particles gets more and more difficult. At $10 \mathrm{AU}$ in the disk, it is still possible to form mm-sized particles 


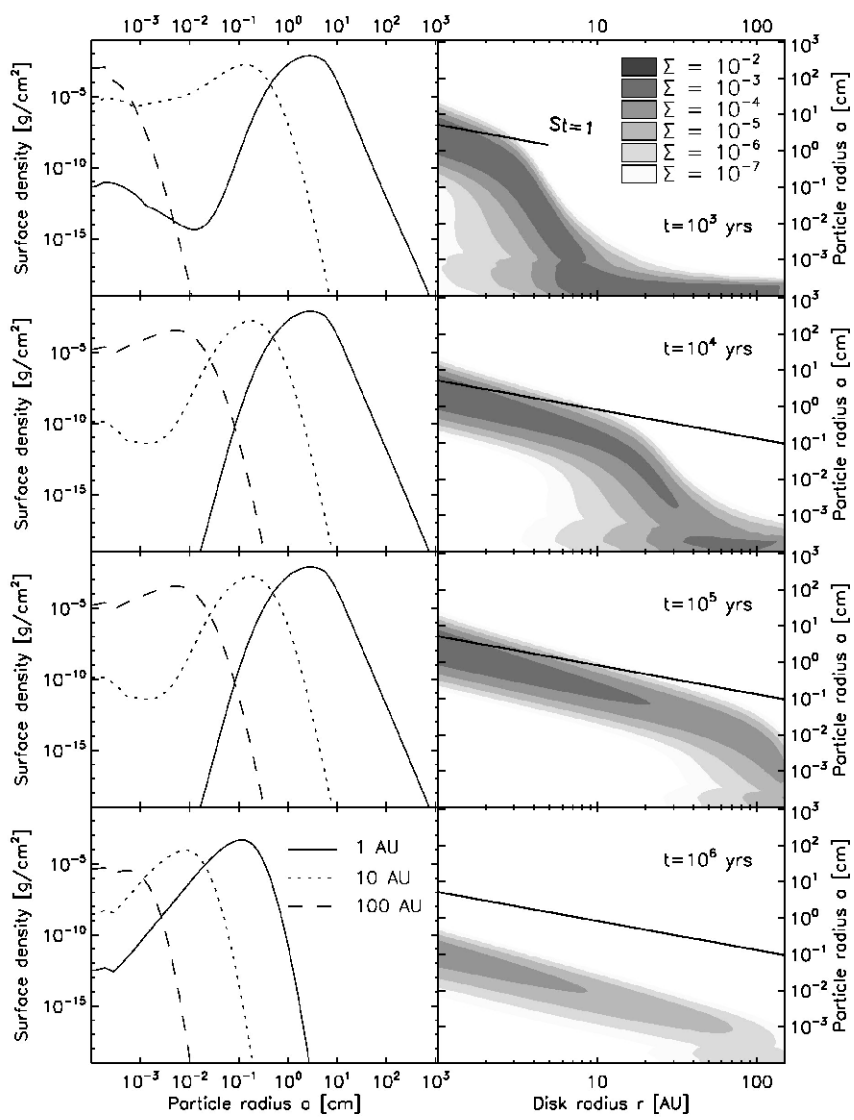

Fig. 7. The particle size distribution at different radii in the disk at different times of disk evolution as discussed in Sect. 3.2.1. In this simulation all particle gowth mechanisms are included as well as the radial motion of the dust. The fragmentation of particles is neglected. The left and the right plots always belong together. The left column shows the surface density as a function of particle radius at 1,10 and $100 \mathrm{AU}$. The right column shows the corresponding contour plots of the surface density as a function of disk radius and particle radius. The white lines in the contour plots denotes the particle radius for which the Stokes number is unity (i.e. largest radial drift and largest radial velocities).

in $10^{4}$ yrs according to Fig. 7. However, in the outer parts of the disk ( $>100 \mathrm{AU})$ the dominant particle size of the dust never exceeds $0.1 \mathrm{~mm}$ at any time. The disregard of radial drift in the previous section led to particle sizes of more than a centimeter at $100 \mathrm{AU}$ after $1 \mathrm{Myr}$, which is orders of magnitude larger.

The neglect of radial drift, as discussed in Sect. 3.1, involved a permanent amount of small particles which was present throughout the disk for at least $1 \mathrm{Myr}$. These small particles were located high above the midplane and were subject to a rather slow coagulation process due to relatively low dust densities. Figure 7 indicates that there is a smaller remaining amount of small dust if radial motion is taken into account. This is due to the following reason. Even the small particles in the higher regions of the disk can have relative radial velocities of the order of some $\mathrm{mm} / \mathrm{s}$ or even $\mathrm{cm} / \mathrm{s}$. These higher relative velocites lead to higher collision rates and, hence, to a depletion of the small dust grains.

After $10^{5}$ yrs of disk evolution, the average particle size at a certain radius in the disk starts to decrease in time. To give an example, after $10^{5} \mathrm{yrs}$ the dominant dust grain radius at $1 \mathrm{AU}$ is $\sim 1 \mathrm{~cm}$. After $1 \mathrm{Myr}$ this value is about an order of magnitude lower. While particles drift inward from a certain radial position they are replaced by other particles from the outer parts of the

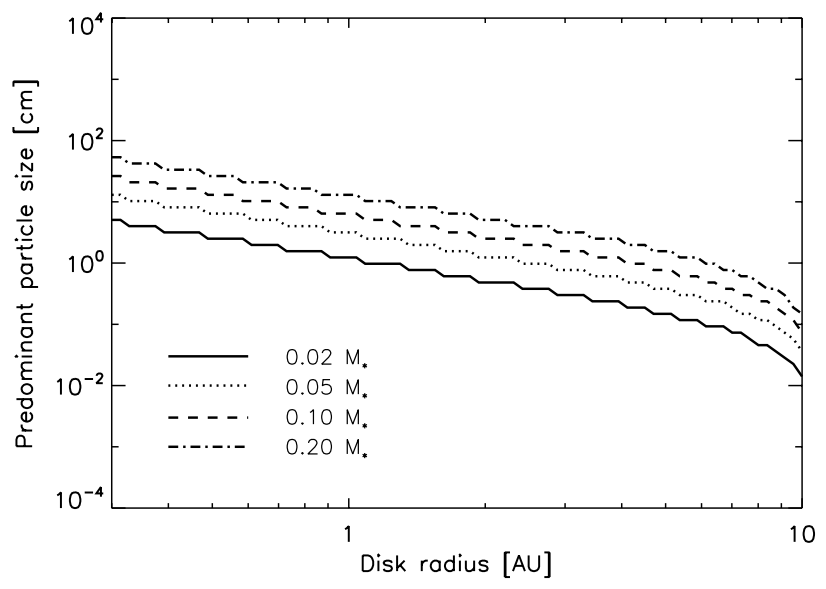

Fig. 8. The effect of disk mass on the particle growth as discussed in Sect. 3.2.2. Shown is the dominant dust particles radius after $10^{4} \mathrm{yrs}$ of disk evolution for different disk masses between 0.2 and $10 \mathrm{AU}$. The turbulent $\alpha$ parameter is $10^{-3}$ and the initial dust-to-gas ratio is $10^{-2}$.

disk. The coagulation time scales are larger in the outer parts of the disk which means that particles grow to smaller sizes in the same time. Therefore, the particles that reach a certain position are smaller than the particles that drift away and, hence, the average dust particle size decreases.

In the simulation shown in Fig. 7 the Stokes number of the dominant particles never exceeds unity, i.e. never breaks the radial drift barrier, at any disk radius considered at any time. This is indicated by the $\mathrm{St}=1$ line which is also shown in this plot. At $\sim 1 \mathrm{AU}$ in the disk, the simulation shows that particles may grow to sizes that correspond to a Stokes number slighly smaller than unity. In the following we will investigate if the particles may break through the $\mathrm{St}=1$ barrier for certain disk parameters.

\subsubsection{Effect of disk mass}

We investigate the effect of disk mass on the particle growth. The result of this investigation can be seen in Fig. 8. This plot shows the dominant dust particle size for different disk masses after $10^{4} \mathrm{yrs}$ of disk evolution as a function of disk radius. We find that the particle size increases by an order of magnitude if the disk mass is increased from $1 \%$ to $20 \%$ of the central mass. Larger disk masses lead to higher gas and dust densities and, hence, to higher collision rates according to Eq. (24). Therefore, dust particles can grow to larger sizes over the same time interval.

The Stokes number of the dominant particles is always smaller than unity. Of course, particles may grow to larger sizes which increases the Stokes number since St $\propto a$. However, larger disk masses also lead to higher gas densities which again decreases the Stokes number because St $\propto 1 / \rho_{\mathrm{g}}$. Finally, both effects cancel out and the disk mass seems to plays a minor role in breaking the radial drift barrier.

\subsubsection{Effect of turbulence}

As in the last section, we calculate the dominant particle size after $10^{4}$ years but now for different turbulent $\alpha$-parameters instead of different disk masses. The initial dust-to-gas ratio in this simulation is $10^{-2}$, the disk mass is $10^{-2} M_{\star}$ and the result is shown in Fig. 9.

One would intuitively think that in a certain time particles can grow to larger sizes in highly turbulent disks than in lowturbulent disks. Figure 9 shows, however, that the dominant 


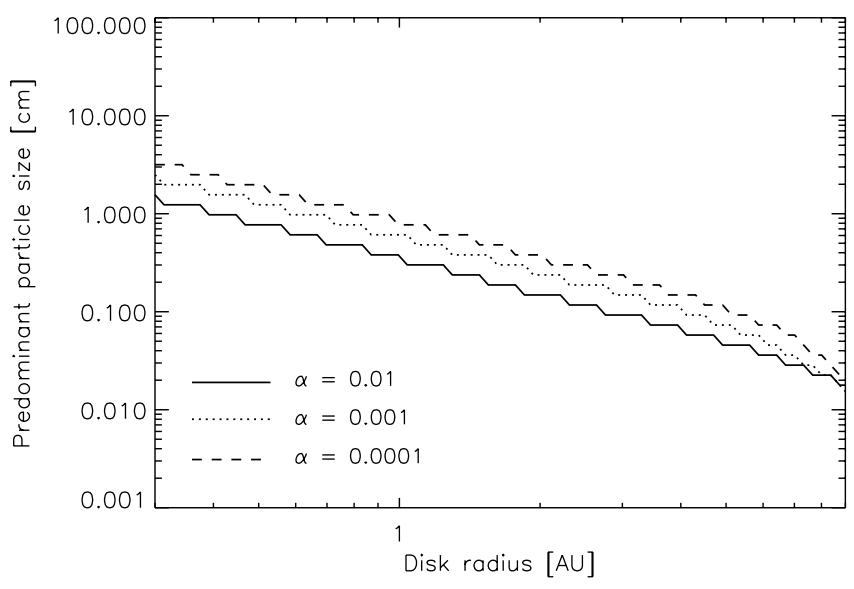

Fig. 9. Same plot as Fig. 8 but now showing the effect of turbulence on the particle growth as discussed in Sect. 3.2.3. Shown is the dominant particles size after $10^{4} \mathrm{yrs}$ of disk evolution for different turbulent $\alpha$ parameters between 0.2 and $10 \mathrm{AU}$. The disk mass is $10^{-2} M_{\star}$ and the initial dust-to-gas ratio is $10^{-2}$.

particle size after $10^{4}$ yrs is only weakly dependent on the turbulence parameter $\alpha$. If $\alpha$ changes by two orders of magnitude then the dominant particle size only changes by a factor of two. This can be understood by the following consideration.

A high amount of turbulence in the disk leads to high relative turbulent particle velocities (Völk et al. 1980; Weidenschilling 1984; Cuzzi et al. 2001). These high relative velocities cause high collision rates, cf. Eq. (24), which favour the process of coagulation. For this reason particles should have grown to larger sizes in highly turbulent disks. On the other hand, a large amount of turbulence leads to thick particle layers. The dust is stirred up in the higher regions of the disk which causes the average dust densities to decrease. The collision rates in Eq. (24) are proportional to the particle number densities. Lower dust particle densities lead to longer coagulation time scales.

The two determining factors for the growth time scales, the relative turbulent particle velocity and the dust density, seem to cancel out if the amount of turbulence in the disk is varied. Hence, different $\alpha$-parameters lead to the same particle size over the same time interval.

\subsubsection{Effect of the initial dust-to-gas ratio}

We now investigate the effect of the initial dust-to-gas ratio on the growth time scales and the particle size distribution. We consider a disk mass of $10^{-2} M_{\star}$ and a turbulence parameter $\alpha$ of $10^{-3}$. The result of this investigation is shown in Fig. 10.

This contour plot shows the surface density of the particle distribution as a function of disk location and particle radius for four different initial dust-to-gas ratios after $10^{4}$ yrs of disk evolution. These results indicate that $10^{4}-10^{5} \mathrm{~cm}$ sized boulders can form in the inner parts of the disk $(<3 \mathrm{AU})$ subject to the condition that the initial dust-to-gas ratio of the disk is higher than $1 \%$. This means that the dust particles may overcome the radial drift barrier if the dust-to-gas ratio is slighly higher than usually assumed. A contour plot of the surface density distribution with $\epsilon_{0}=0.03$, i.e. in the case where the particles are able to break through the radial drift barrier for disk radii $<3 \mathrm{AU}$, as a function of time is shown in Fig. 11.

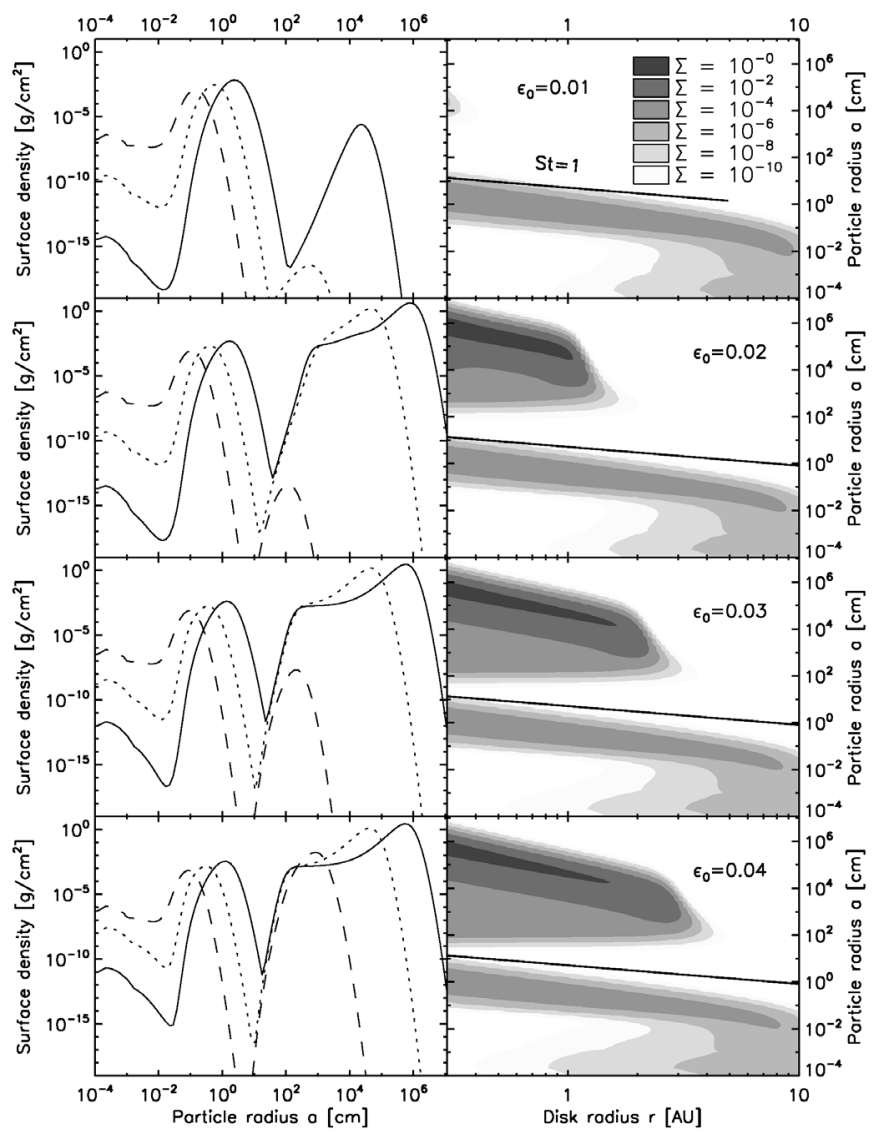

Fig. 10. These plots shows the effect of the initial dust-to-gas ratio on the particle growth as discussed in Sect. 3.2.4. The right side shows contour plots of the surface density as a function of disk location and particle radius for 4 different initial dust-to-gas ratios after $10^{4} \mathrm{yrs}$ of disk evolution. The corresponding left plots show the surface density as a function of particle radius for 3 different locations in the disk (0.3 AU solid, $1 \mathrm{AU}$ - dotted, $3 \mathrm{AU}$ - dashed) after the same time. The disk mass is $10^{-2} M_{\star}$ and the turbulent $\alpha$ parameter is $10^{-3}$. For initial dust-to-gas ratios which are slighly higher than $1 \%$ the particles break through the radial drift barrier.

To understand this importance of the initial dust-to-gas ratio we consider the growth rate of the dust particles as given by Kornet et al. (2001),

$\dot{a}=\frac{\rho_{\mathrm{d}}}{\rho_{\mathrm{s}}} \Delta v$.

If we assume that the particles have Stokes numbers smaller than unity then the relative turbulent particle velocity is given by (Cuzzi et al. 2001; Weidenschilling \& Cuzzi 1993)

$\Delta v_{\text {turb }} \propto \sqrt{\alpha \mathrm{St}} c_{\mathrm{s}}$.

The dust mass density can be approximated by $\rho_{\mathrm{d}} \propto \Sigma_{\mathrm{d}} / h$ so that we obtain

$\dot{a}=\frac{1}{\rho_{\mathrm{s}}} \frac{\Sigma_{\mathrm{d}}}{h} \sqrt{\alpha \mathrm{St}} c_{\mathrm{s}}$

With the height of the dust layer Eq. (15), the last expression can be written as (for $\mathrm{St}<1$ )

$\dot{a}=\frac{1}{\rho_{\mathrm{s}}} \epsilon_{0} \Sigma_{\mathrm{g}} \mathrm{St} \Omega_{\mathrm{k}}$. 


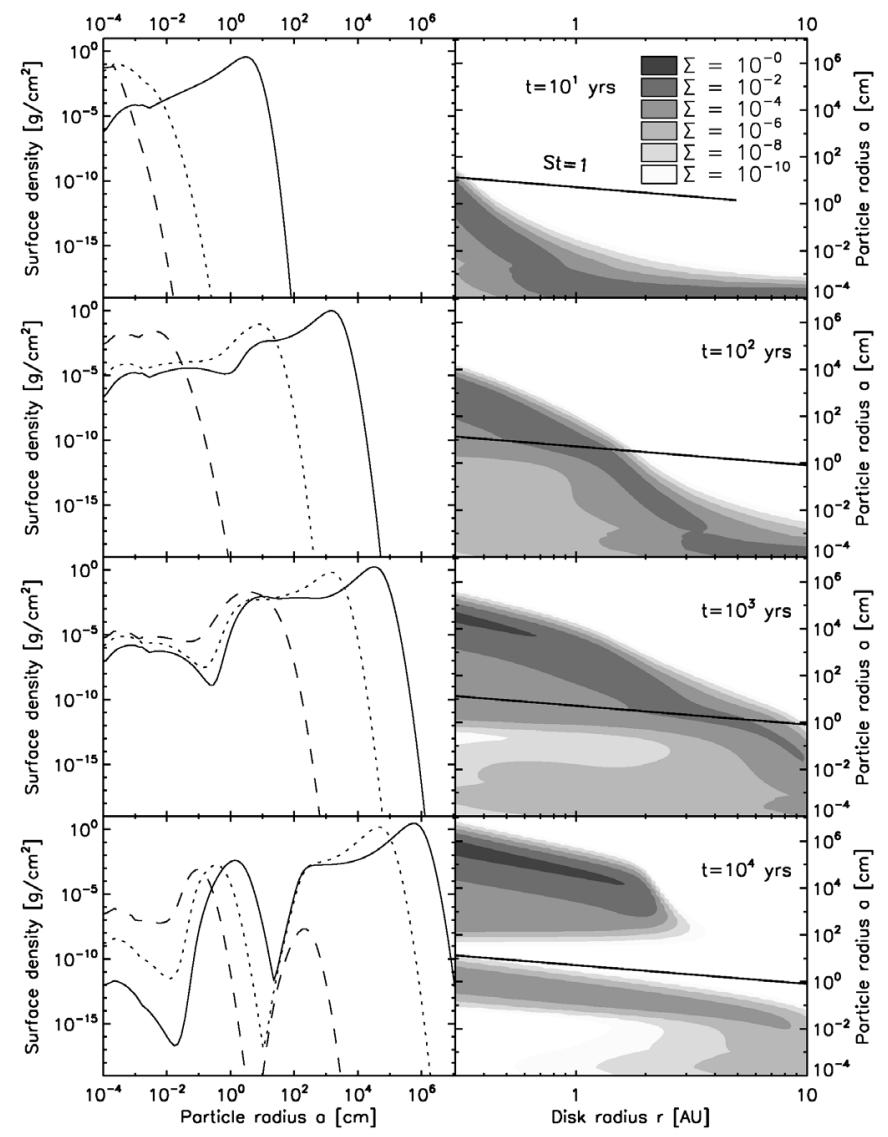

Fig. 11. This plot shows the results of a simulation in which the particles can break through the radial drift barrier as discussed in Sect. 3.2.4. Particle fragmentation is neglected in this simulation. Shown is the surface density distribution for the first $10^{4}$ yrs of disk evolution for an initial dust-to-gas ratio of 0.03 as a function of disk radius and particle radius. The disk mass is $10^{-2} M_{\star}$ and the turbulent $\alpha$ parameter is $10^{-3}$. The right side is a contour plot of the surface density. The left side shows the absolute values of the surface density for 3 different disk radii (solid - 0.3 AU, dotted - $1 \mathrm{AU}$, dashed - $3 \mathrm{AU}$ ).

If we also take into account the definition of the Stokes number in Eq. (13), then most quantities cancel each other out, particularly the gas surface density $\Sigma_{\mathrm{g}}$, leading to

$\dot{a}=a \epsilon_{0} \Omega_{\mathrm{k}}$

with the solution

$a=a_{0} \mathrm{e}^{\epsilon_{0} \Omega_{\mathrm{k}} t}$.

This expression shows that only the initial dust-to-gas ratio $\epsilon_{0}$ and the Kepler frequency $\Omega_{\mathrm{k}}$ determine the turbulent growth time scales as long as $\mathrm{St}<1$. According to Eq. (39), the time scales are not linear dependent on the initial dust-to-gas ratio. An increase of $\epsilon_{0}$ leads to an exponential decrease of the growth time scales. This strong dependency unveils the crucial importance of this initial parameter. Equation (39) also shows that turbulent coagulation occurs faster in the inner parts of the disk than in the outer parts since $\Omega_{\mathrm{k}} \propto r^{-1.5}$. For this reason, the particles first break through the radial drift barrier in the inner parts of the disk (cf. Fig. 10).

In Sects. 3.2.2 and 3.2.3 we have seen that the dominant particle size only shows a weak dependency on the disk mass and the amount of turbulence in the disk. This can also be explained by Eq. (37). The turbulent growth rate of the dust is neither dependent on the disk mass nor on the turbulent $\alpha$ parameter. Moreover, this expression also indicates that the disk temperature and intrinsic particle properties like solid density are rather unimportant as long as the Stokes number of the particles is smaller than unity and turbulence is the leading process that triggers coagulation.

However, Ormel et al. (2007) have shown that the porosity of dust particles actually matters in the early phases of disk evolution. This discrepancy is due to the fact that the Eq. (39) only holds if St $>\alpha$ while Ormel et al. (2007) considered particles with $\mathrm{St}<\alpha$. Moreover, Brownian motion is the main source for relative particle velocities for small dust grains in the early disk evolution while the derivation of Eq. (39) assumes that turbulence is the major source for relative particle velocities.

\subsubsection{The radial drift barrier}

Now we will estimate in which regions of the disk and under which conditions the solid particles can theoretically overcome the radial drift barrier.

In Sect. 3.2.4 we have seen that particle coagulation due to turbulence in the disk can be described by $\dot{a}=a \Omega_{\mathrm{k}} \epsilon_{0}$. We define a particle growth time scale $\tau_{\mathrm{g}}$ by

$\tau_{\mathrm{g}}=\gamma \frac{a}{\dot{a}}=\frac{\gamma}{\Omega_{\mathrm{k}} \epsilon_{0}}$.

The parameter $\gamma$ measures how much the solid particle has to grow to cross the particle size region of fast radial drift, i.e. to overcome the radial drift barrier. We assume this parameter to have a certain value determined by the disk model and to be a constant throughout the disk. The largest radial drift velocity in the disk is approximately given by $c_{\mathrm{s}}^{2} / V_{\mathrm{k}}$. We define a radial drift time scale $\tau_{\mathrm{d}}$ by

$\tau_{\mathrm{d}}=\frac{r}{c_{\mathrm{s}}^{2} / V_{\mathrm{k}}}$

The ratio between these two time scales is given by

$\frac{\tau_{\mathrm{g}}}{\tau_{\mathrm{d}}}=\frac{\gamma}{\epsilon_{0}}\left(\frac{H}{r}\right)^{2}$

In the last step we made use of Eq. (15). Now, the particles may overcome the radial drift barrier if the ratio $\tau_{\mathrm{g}} / \tau_{\mathrm{d}}$ is smaller than unity, i.e. if the growth time scales are smaller than the radial drift time scales. The parameter $\gamma$ is still indefinite.

To specify this parameter we consider Fig. 10 in Sect. 3.2.4. These simulation results show for which initial dust-to-gas ratio $\epsilon_{0}$ the particles break through the meter size barrier at a certain radius in the disk. We chose the parameter $\gamma$ in a way that the condition $\tau_{\mathrm{d}}>\tau_{\mathrm{g}}$ is in agreement with the results shown in this figure. This leads to $\gamma \approx 12$. With this value, the particles should overcome the radial drift barrier if the inequality

$\epsilon_{0} \gtrsim 12\left(\frac{H}{r}\right)^{2}$

holds.

The particles, which break through the radial drift barrier in Fig. 10, have already drifted inwards. For this reason, the critical value given by Eq. (43) indicates the initial dust-to-gas ratio for which the particles most likely break through the radial drift barrier. The sufficient $\epsilon_{0}$-value to overcome the radial drift barrier is presumably even lower than this value. 


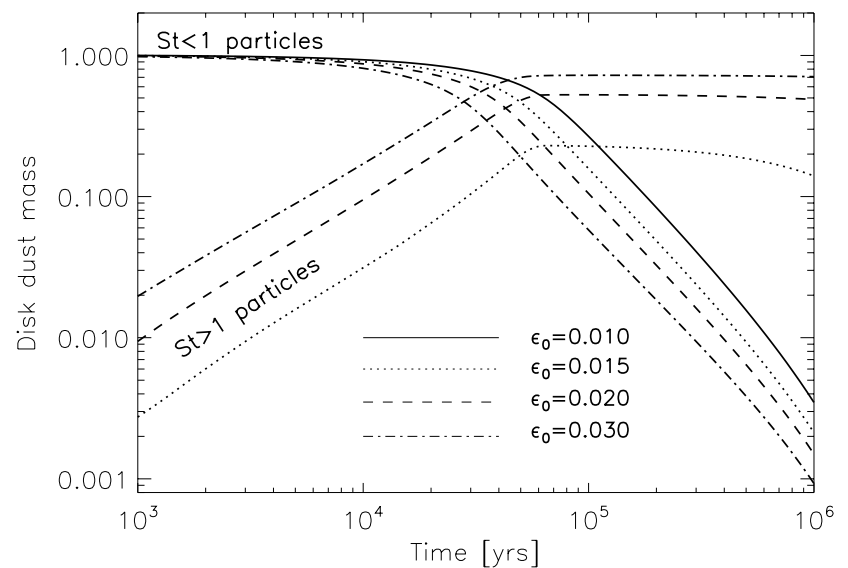

Fig. 12. The mass of the dust disk for small $(\mathrm{St}<1)$ and large $(\mathrm{St}>1)$ particles between $0.5 \mathrm{AU}$ and $150 \mathrm{AU}$ as a function of time for different initial dust-to-gas ratios as discussed in Sect. 3.2.6. In this simulation only the particle coagulation and the radial motion of the dust were considered. Particle fragmentation was neglected.

\subsubsection{Dust mass loss in the disk}

When particles drift into the evaporation zone, they are lost for the process of planetesimal formation. Hence, the question of how much solid material is actually lost due to its drift into the inner regions is of essential importance. We calculate the mass which is present in small $(\mathrm{St}<1)$ and large $(\mathrm{St}>1)$ particles between $0.5 \mathrm{AU}$ and $150 \mathrm{AU}$ as a function of time for different initial dust-to-gas ratios. The result of this calculation can be seen in Fig. 12.

This plot shows, that the mass of the dust disk does not change significantly within the first $10^{4}$ yrs for every initial dustto-gas ratio considered. Since the power law index of the surface density is -0.8 , most of the solid particles are in the outer regions of the disk. Fast radial drift in the inner disk regions which takes place in $\sim 10^{3}$ yrs does not change the total dust mass in the disk.

After a few $10^{4}$ yrs, the mass present in small grains starts to decrease. After $1 \mathrm{Myr}$ of disk evolution, this mass is less than $1 \%$ of the initial dust mass. The amount in small grains, i.e. St $<1$ particles, is dependent on the initial dust-to-gas ratio. For $\epsilon_{0}=0.01$ roughly $0.4 \%$ of the initial particle mass is present in small grains. For $\epsilon_{0}=0.03$ this mass is a factor of 4 lower. Hence, higher initial dust-to-gas ratios lead to lower dust masses in small grains after $1 \mathrm{Myr}$. In the last section we showed that particles grow faster with increasing dust-to-gas ratio. Therefore, particles can grow to larger sizes while moving radially inwards. However, larger sizes also lead to higher radial drift velocities (Eq. (17)). For this reason, the mass which is present in small particles in the disk decreases faster for increasing dust-to-gas ratios. We will find the same behaviour for the small particles in step 3 where fragmentation is also taken into account.

For an initial dust-to-gas ratio of $1 \%$, the mass of the entire dust disk, i.e. the mass in small and large particles, after 1 Myr between $0.5 \mathrm{AU}$ and $150 \mathrm{AU}$ is $0.4 \%$ of the initial dust mass. Most of the dust has drifted into the evaporation zone. For higher initial dust-to-gas ratios, i.e. higher than 0.015 , the particles in the inner regions of the disk can break through the radial drift barrier. These larger boulders around 1 AU then sweep up smaller particles which drift inwards from larger radii (cf. Fig. 12 between $10^{3}$ yrs and $\sim 5 \times 10^{4} \mathrm{yrs}$ ). After $\sim 5 \times 10^{4} \mathrm{yrs}$ most of the dust mass is present in large boulders. While for $\epsilon_{0}=0.015$ roughly $20 \%$ of the initial dust mass is present in
St $>1$ particles after $1 \mathrm{Myr}$, the remaining mass in large boulders is a factor of $\sim 4$ higher for $\epsilon_{0}=0.03$. Note that the mass of the remnant dust disk after $1 \mathrm{Myr}$ changes by a factor of $\sim 200$ by changing the initial dust-to-gas ratio from $1 \%$ to $3 \%$. We conclude that the initial dust-to-gas ratio is a crucial parameter which has an important influence on how much solid material remains in the disk after 1 Myr. However, the mass present in small grains is always less than $0.4 \%$ of the initial dust mass after 1 Myr no matter the value of $\epsilon$.

\subsection{Step 3 - Coagulation, radial motion and fragmentation}

We now also include particle fragmentation in our simulation. We investigate how this destructive effect influences the particle growth in the disk and how various disk parameters, like the initial dust-to-gas ratio, the turbulence parameter $\alpha$ or the fragmentation velocity $v_{\mathrm{f}}$, influence the coagulation/fragmentation process.

\subsubsection{Time evolution}

The evolution of the disk in the first 1 Myr is shown in Fig. 13. In this calculation, the fragmentation velocity is $v_{\mathrm{f}}=10^{3} \mathrm{~cm} / \mathrm{s}$ and the fragmentation parameter $\xi$ is 1.83 . We adopt a disk mass of $10^{-2} M_{\star}$, a turbulent $\alpha$-value of $10^{-4}$ and an initial dust-to-gas ratio of $10^{-2}$. The cratering-parameter $\chi$ is 0.5 and $\psi=2$.

After $10^{3}$ yrs of disk evolution, most of the particles in the disk $<3$ AU have grown to sizes of some millimeters. However, if fragmentation is neglected (cf. Sect. 3.2) the dominant particle size at $1 \mathrm{AU}$ in the disk after $10^{3} \mathrm{yrs}$ is an order of magnitude larger. This significant difference is due to the fragmentation of particles. When the particles reach millimeter size then destructive effects prevent the particles from growing to larger sizes (cf. Fig. 3 with $10 \mathrm{~m} / \mathrm{s}$ ). Even after $10^{4} \mathrm{yrs}$, the dominant particle size in the disk $<10 \mathrm{AU}$ is still of the order of a millimeter. Hence, this particle size corresponds to the fragmentation barrier for this specific set of disk parameters. Even for long periods of time the particles are not able to overcome this barrier. Once the particles have reached the fragmentation barrier the particle distribution is characterised by an equilibrium between particle coagulation and particle fragmentation due to destructive collisions. In other words, the amount of particles of a certain mass, which are created by dust particle coagulation, equals the amount of particles, which are destroyed by high velocity collisions. This steady state will be discussed in more detail later in this section.

Figure 13 indicates that the maximum dominant particle size $a_{\max }$ and the Stokes number St have the same radial behaviour. This is due to the fact that relative particle velocities in our model (except Brownian motion) scale with this dimensionless number. For this reason, the dominant particle size follows $a_{\max } \propto r^{-0.8}$ which we obtain directly from the definition given by Eq. (13).

Due to destructive collisions a large amount of dust is present in small grains as can be clearly seen in Fig. 13. We calculate the amount of dust which is present in grains larger (smaller) than $10^{-2} \mathrm{~cm}$ after $10^{5}$ yrs of disk evolution. While $18 \%$ of the dust mass is present in grains larger than $10^{-2} \mathrm{~cm}$, yet $82 \%$ of the mass is present in smaller grains. This large population of sub$\mathrm{mm}$ grains should have a strong effect on the spectrum of the protostellar disk. However, we will not investigate the influence of the fragmentation parameters, i.e. $v_{\mathrm{f}}$ and $\xi$, on the disk 


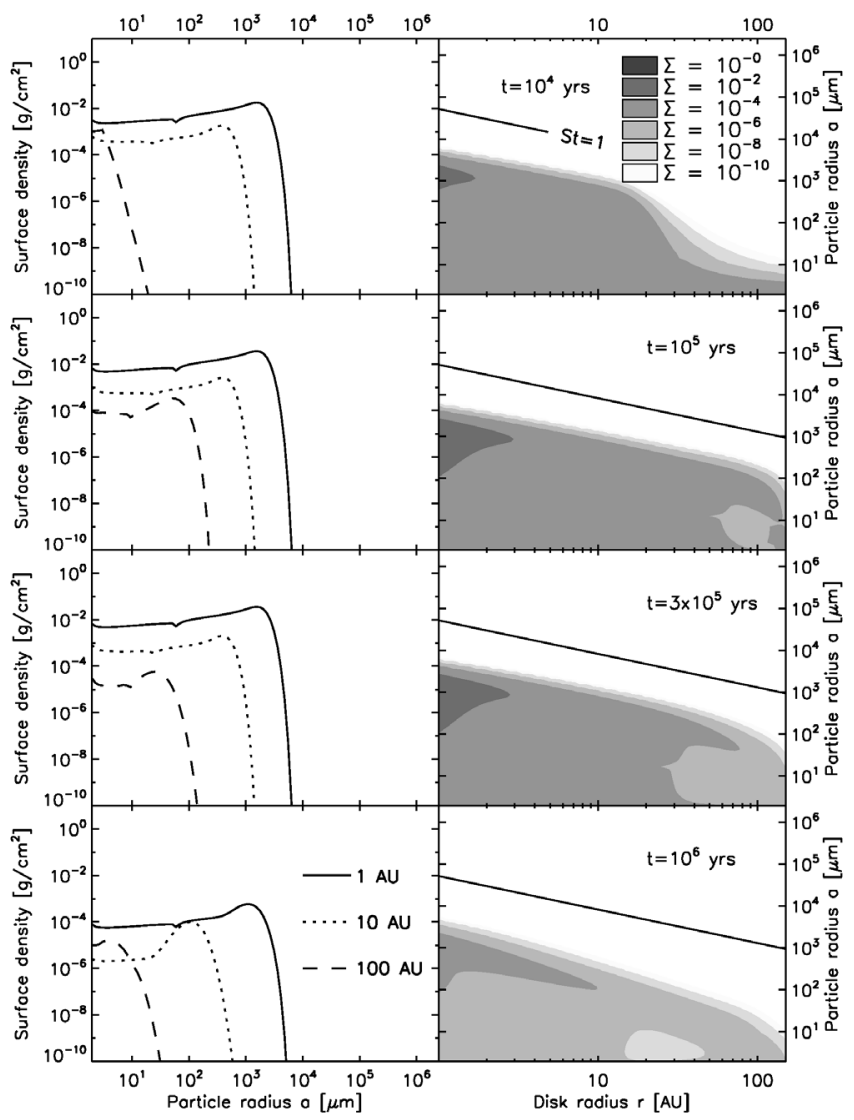

Fig. 13. As Fig. 7, but now also the fragmentation of particles is included in the simulations as discusssed in Sect. 3.3.1. The left column shows the surface density as a function of particle radius at 1,10 and $100 \mathrm{AU}$. The right column shows the corresponding contour plots of the surface density as a function of disk radius and particle size.

spectrum which goes beyond the scope of this paper. This will be investigated in the near future.

\subsubsection{Effect of turbulence}

Different turbulent $\alpha$-values should lead to different maximum particle sizes due to destructive collisions. To investigate the influence of turbulence on the fragmentation barrier, we calculate the dominant particle size for different $\alpha$-values after $10^{4} \mathrm{yrs}$ of disk evolution. In this simulation the disk mass is $10^{-2} M_{\star}$, the fragmentation velocity is $10^{3} \mathrm{~cm} / \mathrm{s}$, the initial dust-to-gas ratio is $10^{-2}$ and the results of the calculation are shown in Fig. 14.

According to this plot, the dominant particle size is fairly dependent on $\alpha$ in moderately turbulent disks. If $\alpha$ is changed from $10^{-3}$ to $10^{-4}$ then the dominant particle size $a_{\text {dom }}$ changes by a factor of $\sim 5$. We find that less turbulence shifts the fragmentation barrier towards larger particle sizes. Hence, in less turbulent disks particles can grow to larger sizes than in highly turbulent disks.

However, this statement does not hold for extremely low turbulent disks. In these disks, turbulence is not the main source for relative velocities and, hence, the fragmentation barrier should not be dependent on $\alpha$. If $\alpha$ is smaller than $\sim\left(c_{\mathrm{s}} / 2 V_{\mathrm{k}}\right)^{2}$ (cf. Eqs. (18) and (35)) which is $\sim 10^{-4}$ at $1 \mathrm{AU}$ then relative particle velocities due to radial motion exceed relative dust particle motions induced by turbulence. To illustrate this independency we calculate the dominant particle size after $10^{4} \mathrm{yrs}$ for a disk with a very low $\alpha$-value of $10^{-10}$. The result of this calculation

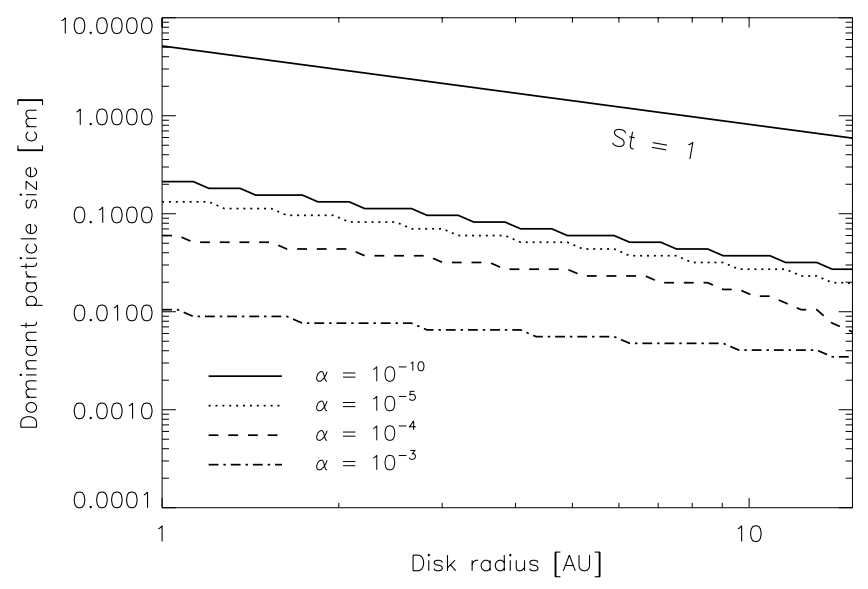

Fig. 14. The influence of the turbulence parameter $\alpha$ on the dominant particle size after $10^{4} \mathrm{yrs}$ of disk evolution for different disk radii between 1 and $20 \mathrm{AU}$ as discussed in Sect. 3.3.2. The disk mass is $10^{-2} M_{\star}$, the fragmentation velocity is $10^{3} \mathrm{~cm} / \mathrm{s}$ and the initial dust-togas ratio is $10^{-2}$. This graph also shows the particle size for which the Stokes number ist unity. The $\chi$ parameter is set to 0.5 and $\psi=1$.

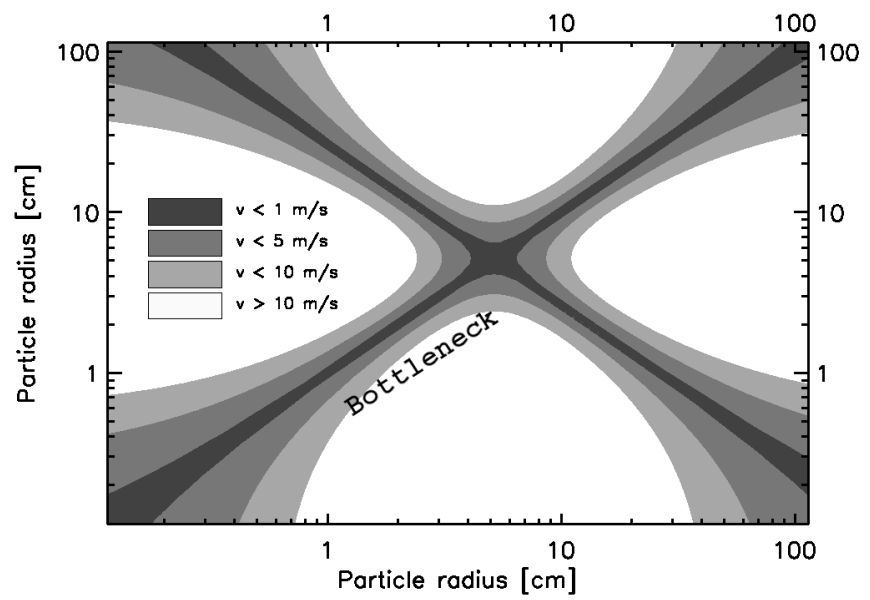

Fig. 15. The relative particle velocities at $1 \mathrm{AU}$ as a function of particle radius as discussed in Sect. 3.3.2. The turbulence parameter $\alpha$ in this calculation is $10^{-10}$. This means that relative radial motion is the main source for relative particle velocities. A critical fragmentation velocity of $10 \mathrm{~m} / \mathrm{s}$ results in a very narrow band in which particle coagulation is still possible. If the particle size dispersion is larger than the extent of this bottleneck then particle fragmentation starts to play a non-negligible role.

is also shown in Fig. 14. In this nearly laminar disk, destructive collsions due to relative drift velocities up to $50 \mathrm{~m} / \mathrm{s}$ prevent particle growth to sizes of more than $\sim 2 \mathrm{~mm}$ at $1 \mathrm{AU}$.

Relative radial drift velocities are always due to particle size differences. Monodisperse distributions do not show relative radial motion. The simulation result for extremely low turbulent disks $\left(\alpha=10^{-10}\right)$ raises the question how the particle size dispersion of the dust distribution can produce such high relative velocities to inhibit particle growth to larger sizes ${ }^{2}$.

We try to answer this question by considering the relative velocities of dust particles at $1 \mathrm{AU}$ in the disk as a function of particle radius, cf. Fig. 15. In this calculation, we adopt an $\alpha$-value of $10^{-10}$ which means that relative radial motion is the main source for relative velocities. According to this figure, particle

\footnotetext{
${ }^{2}$ We define the particle size dispersion as the half-width of the size distribution.
} 


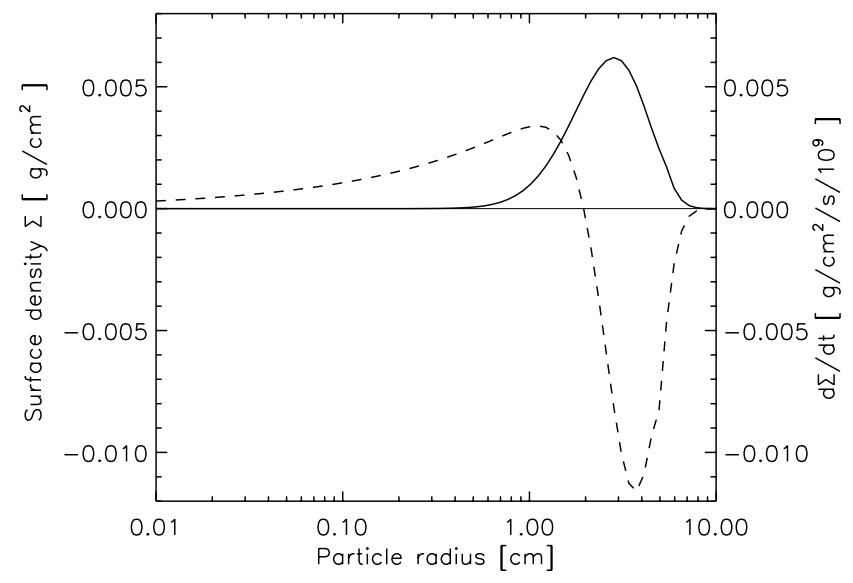

Fig. 16. The dust particle distribution (solid) and its time derivative (dashed) as a function of particle size after $700 \mathrm{yrs}$ of evolution at $1 \mathrm{AU}$ in the disk as discussed in Sect. 3.3.2. The distribution is located around $a_{0}=3 \mathrm{~cm}$ and it has a size dispersion of $\sim 1.5 \mathrm{~cm}$. In these $700 \mathrm{yrs}$, particle fragmentation was neglected. In the calculation of the source terms, which are shown in this figure, fragmentation is included. These source terms show that destructive collsions would rapidly shift the dominant particle size to smaller values.

coagulation is only possible in a very narrow particle size interval, i.e. in the dark shaded regions of this plot. If the particle size dispersion is larger than the extent of this "bottleneck" then particle fragmentation starts to play a non-negligible role. With Eq. (20) for the radial velocities, we can estimate the importance of fragmentation for a specific particle size dispersion. We assume a particle size distribution which has a surface density maximum at $a_{0}=3 \mathrm{~cm}$. If the size dispersion is larger than $1 \mathrm{~cm}$, then particles start to fragment with $100 \%$ probability. For a particle size dispersion of $0.5 \mathrm{~cm}$ and $0.1 \mathrm{~cm}$, the fragmentation probability decreases to $50 \%$ and $10 \%$, respectively. Hence, only for particle size dispersions of some millimeters, particles might have the chance to overcome the fragmentation barrier. For larger size dispersions, the fragmentation probability is far too high to allow the distribution to pass the bottleneck shown in Fig. 15.

To investigate if the particle size dispersion is narrow enough to overcome the fragmentation barrier, we consider the following. We simulate $700 \mathrm{yrs}$ of dust particle evolution neglecting fragmentation. The result of this simulation, i.e. the particle distribution at $1 \mathrm{AU}$ in the disk as a function of particle size, is shown in Fig. 16 (solid line). The size dispersion of this particle distribution is $\sim 1.5 \mathrm{~cm}$. Now, particle fragmentation tends to smear out the dust distribution and it increases the particle size dispersion. Hence, the distribution shown is Fig. 16 represents a best case scenario; the distribution can not become narrower. What happens if we now switch on fragmentation? Figure 16 also shows the time derivative of the particle distribution (dashed line) if fragmentation is considered. This curve indicates, that destructive collsions would rapidly shift the dominant particle size towards smaller values. The size dispersion is apparently too large for the particles to pass through the fragmentation bottleneck without undergoing substantial destructive collsions. If fragmentation is included in the simulations from the very beginning, then the size dispersion is even larger and, therefore, the chance of passing the narrow region of coagulation becomes even smaller.

For a fragmentation velocity of $10 \mathrm{~m} / \mathrm{s}$, which we adopt in these simulations, the particles never overcome the fragmentation barrier, regardless of the amount of turbulence in the disk

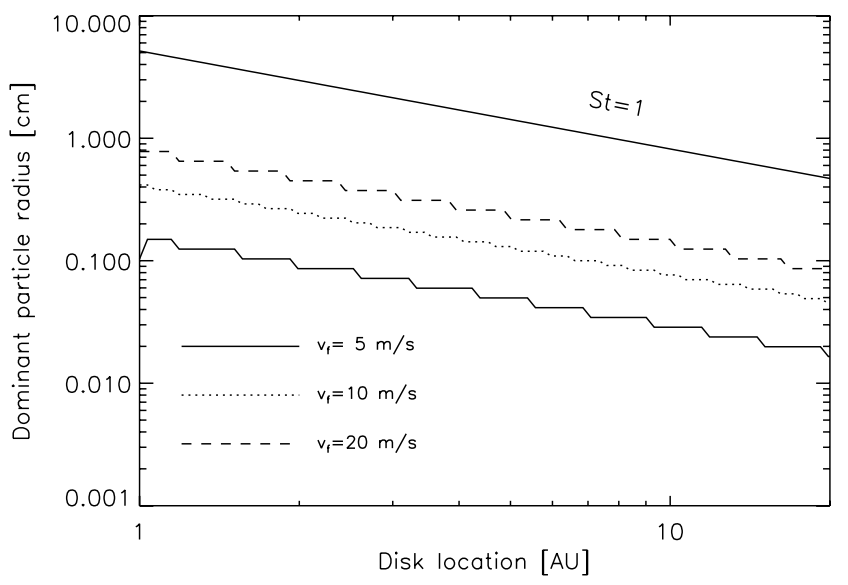

Fig. 17. The dominant particle size as a function of disk location for 3 different fragmentation velocities after $10^{4}$ yrs of disk evolution as discussed in Sect. 3.3.3. In this simulation, $\psi=2, \chi=0, \epsilon_{0}=0.03$ and the turbulent $\alpha$-value is $10^{-5}$.

since the radial drift always accounts for destruction. We find that this statement also holds for larger $\psi$-values. We conclude that the amount of turbulence in the disk alone does not determine whether particles can break through the fragmentation barrier or not. Note, that the maximum radial drift velocity of particles is independent of radius, so that these statements hold everywhere in the disk.

There are possible scenarios in which the radial particle velocity, which is the main reason for particle fragmentation in low turbulent disks, is lower than in the model discussed here. In these cases, particles might overcome the fragmentation barrier. One possibility are local gas pressure fluctuations. Since the radial drift velocity is proportional to the radial gas pressure gradient, local gas maxima can slow down and even prevent radial particle motion. Therefore, we expect dust coagulation instead of dust fragmentation in these maxima. Also local dust particle enhancements can slow down radial drift. Johansen et al. (2006) have shown that the radial drift velocity can be reduced by a factor of around 2. Further investigations of particle growth under these conditions, which make particle fragmentation less likely, are needed.

\subsubsection{Effect of the fragmentation velocity}

For which critical fragmentation velocities can particles break through the fragmentation barrier? To answer this question let us consider a best case scenario. We adopt a low turbulent disk, i.e. a disk in which the relative radial velocities exceed the relative turbulent particle velocities, and we neglect the effect of cratering for the moment. We calculate the dominant dust particle size as a function of disk location for 3 different fragmentation velocities after $10^{4}$ yrs of disk evolution. The results of this calculation can be seen in Fig. 17. In this simulation, the $\alpha$-value is $10^{-5}, \chi=0$ (no cratering) and $\psi=2$.

For a fragmentation velocity of $5 \mathrm{~m} / \mathrm{s}$, particles can grow to millimeter size at $\sim 1$ AU in the disk before destructive collisions prevent further particle growth. In the outer regions, i.e. at $10 \mathrm{AU}$, the dominant particle radius is a factor of 10 smaller. Even for a relatively high critical velocity of $20 \mathrm{~m} / \mathrm{s}$ the particles are not able to grow beyond a centimeter at $1 \mathrm{AU}$.

For even higher fragmentation velocities, i.e. $v_{\mathrm{f}} \sim 30 \mathrm{~m} / \mathrm{s}$, solid particles start to break through the fragmentation barrier. Figure 18 shows the dust particle distribution for this critical 


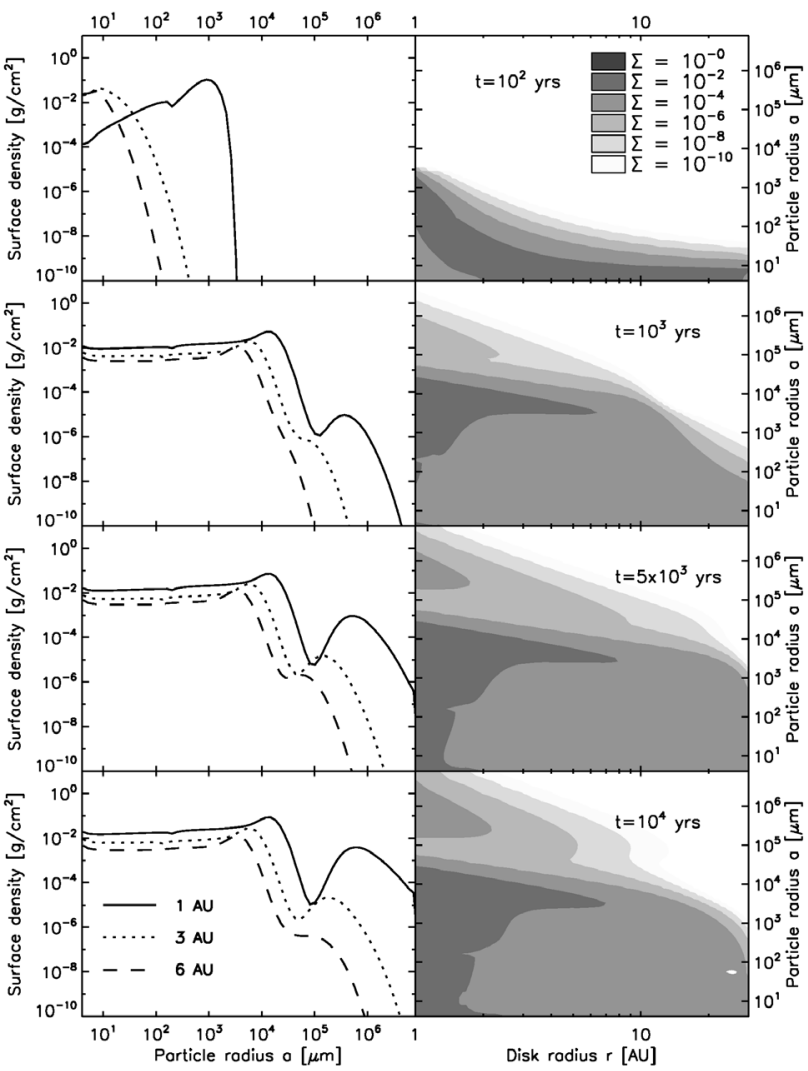

Fig. 18. These plots show how the particles break through the radial drift barrier and the fragmentation barrier as discussed in Sect. 3.3.3. Shown are contour plots of the surface density as a function of disk radius and particle radius at 4 different times of disk evolution. The fragmentation velocity is chosen to have the relatively high value of $30 \mathrm{~m} / \mathrm{s}$. In this simulation $\psi=2$ and $\chi=0$. The initial dust-to-gas ratio is 0.03 .

velocity as a function of disk radius and particle radius for 4 different times of disk evolution. This plot indicates that particles have grown to meter size in the inner parts of the disk after $10^{4}$ yrs. However, a fragmentation velocity of several $10 \mathrm{~m} / \mathrm{s}$ for centimeter- or even meter-sized boulders is at least questionable. For lower (and probably also more realistic) critical velocities, i.e. velocities of $1 \ldots 10 \mathrm{~m} / \mathrm{s}$, we never find solid particles in our simulations which are able to overcome the fragmentation barrier for any disk parameters considered. For $\alpha$-parameters, which are higher than the adopted value of $10^{-5}$ in the simulations of this paragraph, it is even more unlikely that solid particles may grow to larger sizes. This chance does not increase if destructive effects due to cratering are also taken into account.

\subsubsection{Disk dust mass}

As in Sect. 3.2.6, we calculate the solid material mass in the disk as a function of time, but now with the effect of particle fragmentation included in the simulations. The result of this calculation is shown in Fig. 19.

The dust mass does not change significantly within the first $10^{5} \mathrm{yrs}$ for any $\epsilon_{0}$ considered. This is the same behaviour as in the case of no fragmentation. After $10^{5} \mathrm{yrs}$ the mass starts to decrease rapidly. For an initial dust-to-gas ratio of 0.01 only $2 \%$ of the initial solid material mass between 1 and 150 AU remains after 1 Myr. Higher initial dust-to-gas ratios lead to less solid material after $1 \mathrm{Myr}$. For example, for $\epsilon=0.03$ the mass is only $0.7 \%$ of the initial dust mass, which is a factor of $\sim 3$ lower.

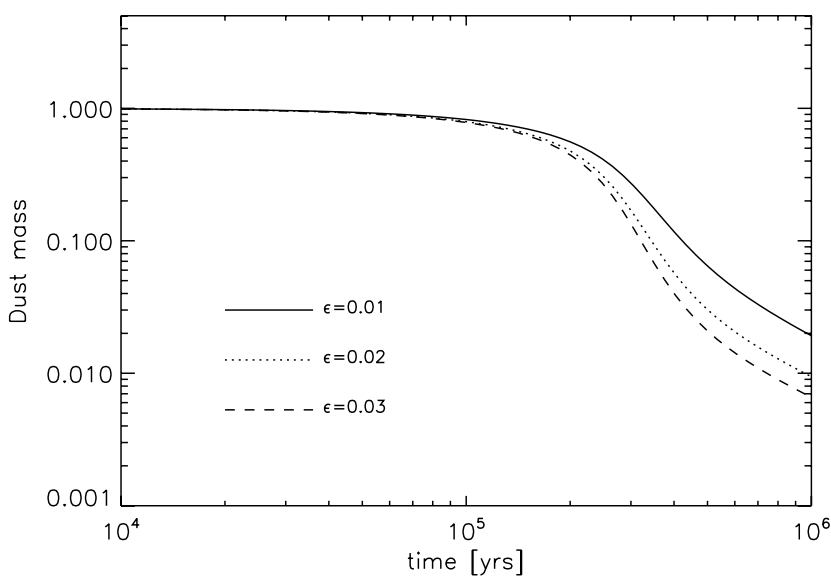

Fig. 19. The mass of the dust disk between $1 \mathrm{AU}$ and $150 \mathrm{AU}$ as a function of time for 3 different initial dust-to-gas ratios as discussed in Sect. 3.3.4. In this simulation, particle growth particle fragmentation and radial motion are included. The initial disk mass of gas and solid material is $10^{-2} M_{\star}, \alpha=10^{-4}, \chi=0.5, \psi=2$ and $v_{\mathrm{f}}=10 \mathrm{~m} / \mathrm{s}$.

Let us compare the solid material mass after 1 Myr for $\epsilon_{0}=0.01$ in the case of fragmentation/no fragmentation. We find that the remaining dust mass is a factor of 5 higher if we allow the particles to destroy each other. This difference is due to destructive collisions which lead to large amounts of small particles in the disk (cf. Sect. 3.3.1). These small dust grains have low radial drift velocities and, hence, long radial drift time scales. In other words, small particles stay much longer in the disk before they evaporate in the inner regions of the disk. For this reason, the solid material mass after $1 \mathrm{Myr}$ is higher in the case of fragmentation than in the case of no fragmentation.

In the previous sections we found that if fragmentation is included in the simulations then the dust particles are not able to break through the meter size barrier. No larger particles in the inner parts of the disk can form which can sweep up smaller dust particles drifting inward from the outer regions. For this reason, most of the solid material after $1 \mathrm{Myr}$ has drifted into the evaporation zone and is lost for the process of planetesimal formation.

\subsubsection{Effect of disk model}

In the introduction we mentioned that the disk model adopted in this paper differs significantly from the MMSN model. This leads to the question of how the results of this paper change if different disk models are considered. In this section, we repeat simulations of Sect. 3.3 with other disk model parameters, attempting to unveil the basic changes in the dust particle distribution. Table 1 shows the disk parameters for the simulations in this section. Model A and B are the MMSN model and the disk model in this paper, respectively. Model C is our model, but now with $10 \%$ disk mass instead of $1 \%$ compared to $M_{\star}$. This leads to gas densities which are comparable to those of the MMSN model. The mass distribution, however, has a much flatter radial dependency. The models $\mathrm{D}$ to $\mathrm{F}$ are the same as $\mathrm{A}$ to $\mathrm{C}$, but with a steeper radial temperature dependency. Andrews \& Williams (2007) observationally find radial temperature profiles with a median power law index of 0.62 . This is slighly higher than the passively irradiated disk profile of 0.5 adopted in our model.

Before we come to the results of the simulations, we will qualitatively discuss the difference between the MMSN model 
Table 1. Disk parameters for the simulations performed in Sect. 3.3.5. The quantity $\beta$ denotes the temperature power law index $T \propto r^{-\beta}$. The Models A and B correspond to the MMSN model and the model adopted in this paper, respectively. Model $\mathrm{C}$ is as the model in this paper but now with $10 \%$ disk mass. The Model $\mathrm{D}$ to $\mathrm{F}$ are as $\mathrm{A}$ to $\mathrm{C}$ but with a slighly steeper radial temperature dependency.

\begin{tabular}{cccc}
\hline \hline Model & $\begin{array}{c}\text { Surface density } \\
\text { power law index } \delta\end{array}$ & $\begin{array}{c}\text { Disk } \\
\text { mass }\end{array}$ & $\begin{array}{c}\text { Temperature } \\
\text { power law index } \beta\end{array}$ \\
\hline $\mathrm{A}$ & 1.5 & 0.01 & 0.50 \\
$\mathrm{~B}$ & 0.8 & 0.01 & 0.50 \\
$\mathrm{C}$ & 0.8 & 0.10 & 0.50 \\
$\mathrm{D}$ & 1.5 & 0.01 & 0.62 \\
$\mathrm{E}$ & 0.8 & 0.01 & 0.62 \\
$\mathrm{~F}$ & 0.8 & 0.10 & 0.62 \\
\hline
\end{tabular}

and the model in the paper at hand. The gas mass densities of our model are generally smaller than those of the MMSN model. This has the following main implications. First, solid particles are less coupled to the motions of the gas. The coupling between the gas and the dust can be described by the Stokes number St, which is given by $\mathrm{St}=\rho_{\mathrm{S}} a / \Sigma$. If the surface density of the gas $\Sigma$ decreases, then St is shifted towards higher values. Therefore, the particle growth barrier due to radial drift and particle fragmentation, which is usually referred to as the "meter size barrier" and which corresponds to the particle radius implied by $\mathrm{St}=1$, is shifted towards lower particle radii. In the MMSN model, particles with a Stokes number of unity have radii of $\sim 2 \mathrm{~m}$ at $1 \mathrm{AU}$ in the disk. A surface density slope of $\delta=0.8$ implies $a \sim 5 \mathrm{~cm}$ for $\mathrm{St}=1$ particles at $1 \mathrm{AU}$. While it seems challenging to grow particles larger than meter in size in the MMSN disk model, it is difficult to grow particles larger than centimeter size in the disk model adopted in the paper at hand.

Second, if the Stokes number is shifted towards higher values then all quantities depending on this number are influenced by this change as well. For example, for Stokes numbers smaller than unity the radial drift velocity of solid particles in the disk is proportional to the Stokes number, $v_{\mathrm{r}} \propto$ St (Weidenschilling 1977a). Now, if the Stokes number is modified due to a change of $\delta$ then also the radial drift of the dust is significantly affected. The Stokes number also determines relative dust particle velocities in turbulent disks and, hence, dust particle growth time scales and the maximum dust particle size due to fragmentation.

Figure 20 shows the particle distribution after 1 Myr of disk evolution for the Models A to F. In these simulations, particle growth, radial particle motion and destructive collisions are included. The initial dust-to-gas ratio is $10^{-2}$ and the $\alpha$-value is $10^{-3}$. The $\psi$-parameter is chosen to be 2 and the cratering parameter $\chi=0.5$. This figure shows that particles can grow to much larger sizes in model A than in model B in the inner parts of the disk. This is due to higher gas densities in the MMSN model which alter the Stokes number and shift the whole particle growth problem towards larger particle radii. At $1 \mathrm{AU}$, the gas density in model $\mathrm{A}$ is a factor of $\sim 15$ higher than in model $\mathrm{B}$. The dominant particle size before fragmentation inhibits further particle growth is $3 \mathrm{~mm}$ in model A and $0.2 \mathrm{~mm}$ in model B. This dominant particle size difference from one model to the other nicely mirrors the gas density difference between the two models. Hence, we find that the dominant particle size is directly proportional to the gas density.

Model $\mathrm{C}$ is the same as the model in our paper (B), but now with $10 \%$ disk mass instead of $1 \%$ compared to $M_{\star}$. Figure 20

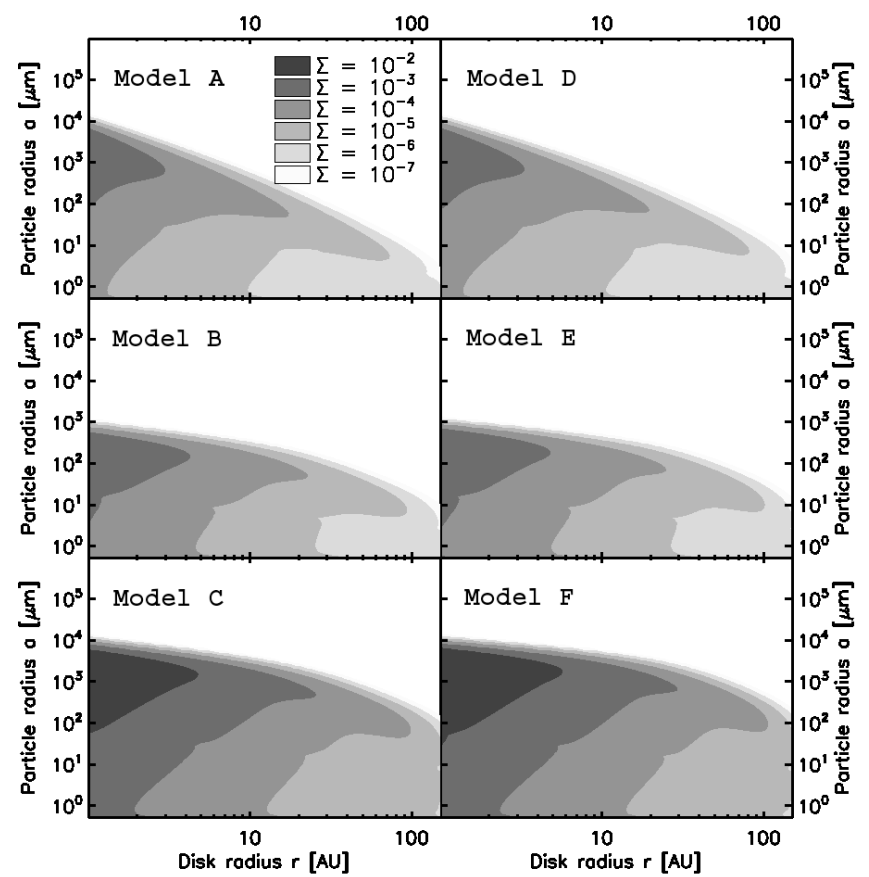

Fig. 20. The particle distribution in the disk after $1 \mathrm{Myr}$ for the disk models A to F as discussed in Sect. 3.3.5. The Models A and B correspond to the MMSN model and the model adopted in this paper, respectively. Model C is as the model in this paper but now with $10 \%$ disk mass. The Model D to F on the right side have a slighly steeper radial temperature dependency.

shows that the dominant particle radius due to destructive collisions is shifted by a factor of 10 towards larger particle sizes. According to these results, particles can grow to a few millimeter in size in high mass disks before particle fragmentation prevents further growth. However, even in these very high mass disks, particles can not overcome the fragmentation barrier. Since the whole coagulation/fragmentation process scales with gas density, higher disk masses do not provide a solution for planetesimal formation. The entire particle growth problem is only shifted towards larger particle radii.

The right column shows the results of the three simulations A-C if the radial temperature dependency follows $T \propto$ $r^{-0.62}$ corresponding to the observational median. We do not find a significant difference in the maximum particle size between these two model sets.

We also calculate the mass of the dust disk which is shown in Fig. 21. This plot shows that the remaining dust mass after $1 \mathrm{Myr}$ of disk evolution is smaller in the MMSN model than in the model adopted in this paper. This is due to the fact that the maximum radial drift velocity is proportional to the power law index $\delta$ of the surface density profile (cf. Eq. (18)). Since the parameter $\delta$ is larger in the MMSN model than in our model, the maximum radial drift speed is also larger. A higher drift speed leads to shorter drift time scales and, hence, reduces the remaining amount of dust after a certain time.

In the disk models D-F, the temperature is generally smaller than in the models A-C. Therefore, the radial drift velocity is also smaller since $v_{n} \propto T$. Hence, the disk dust mass in the model A-C after a certain time is generally smaller than in the models D-F. Finally, we find that less than $6 \%$ of the initial dust mass is left after 1 Myr of disk evolution in any disk model considered. 


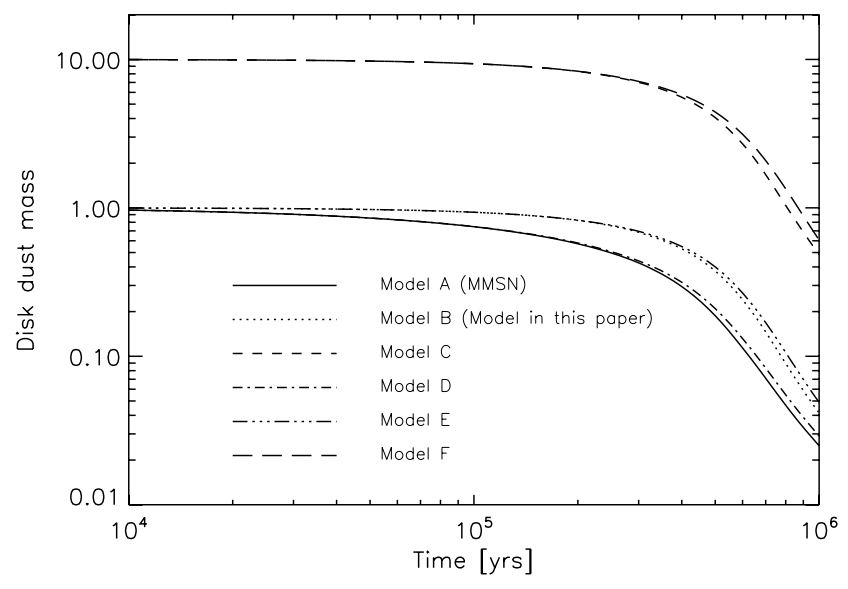

Fig. 21. The mass of the dust disk as a function of time for the 6 different disk models $\mathrm{A}$ to $\mathrm{F}$ as discussed in Sect. 3.3.5. The models with $1 \%$ disk mass are normalised to unity. The disk models with $10 \%$ disk mass are normalised a factor of 10 larger.

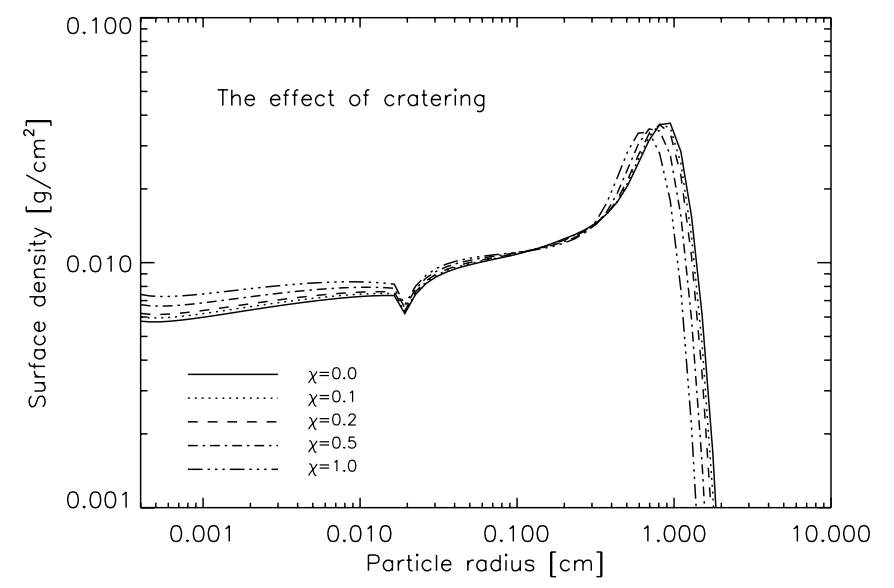

Fig. 22. The effect of cratering on the equilibrium particle distribution as discussed in Sect. 3.3.6. Shown is the surface density of solid particles at $1 \mathrm{AU}$ in the disk after $10^{4} \mathrm{yrs}$ of disk evolution for different crateringparameters $\chi$. In this simulation, the fragmentation velocity is $20 \mathrm{~m} / \mathrm{s}$, $\epsilon=0.03, \alpha=10^{-5}$ and $\psi=2$.

\subsubsection{Effect of cratering}

If a smaller particle of mass $m_{\mathrm{s}}$ collides with a larger body at a sufficiently high velocity then the smaller particle does not only fragment due to this destructive collision but it can also excavate a certain amount of matter $m_{\mathrm{c}}$ from the larger body, i.e. $m_{\mathrm{c}}=\chi m_{\mathrm{s}}$. This effect is called cratering. In the following we investigate if this process has an effect on the equilibrium particle distribution between particle coagulation and particle fragmentation.

Figure 22 shows the equilibrium particle distribution at $1 \mathrm{AU}$ in the disk after $10^{4}$ yrs of disk evolution for different crateringparameters $\chi$. The fragmentation velocity is $20 \mathrm{~m} / \mathrm{s}, \epsilon=0.03$, $\alpha=10^{-5}$ and $\psi=2$.

This plot shows that the equilibrium distribution is hardly affected whether the effect of cratering is included in the simulations or not. Changing the $\chi$-value from 0 (no cratering) to 1 (the projectile particle excavates a crater corresponding to its own mass) changes the surface density for $50 \mu \mathrm{m}$-sized dust grains by a factor of 1.3 at most. The maximum peak of the surface density is shifted from $9 \mathrm{~mm}$ to $6 \mathrm{~mm}$ by including cratering. We also investigated the effect of cratering for different fragmentation velocities. In any case we found that cratering does not significantly affect the particle distribution. This shows that the main destruction by fragmentation is due to collisions between particles of not large mass ratio.

For laboratory experiments which investigate the collision of particles for astronomical purposes it is interesting to know which particles collide with which particles in the equilibrium solution discussed in Sect. 3.3.1. For this reason, we calculate collision rates between dust particles in the disk in Appendix E and we show which collisions are most important for growth/fragmentation.

\section{Discussion and conclusions}

In this paper we study the evolution of the dust in a protoplanetary disk. We expanded on the work done by Dullemond \& Dominik (2005) by adding an improved treatment of dust aggregate fragmentation and by including the self-consistent radial drift and mixing of dust aggregates. In addition to this, our integration method is faster by orders of magnitude than the original work by Dullemond \& Dominik, by virtue of two new methods. The first of these is the integration of the equations in vertical direction without deleting the vertical structure information, which can be done because the vertical sedimentation-mixing equilibrium is assumed to be known at all times. Secondly, we integrate the coagulation-fragmentation in time using implicit integration, allowing us to overcome the extreme time scale stiffness that prevented Dullemond \& Dominik from performing full scale models including fragmentation over time scales of millions of years.

Using these models we are able to study the problem of the radial drift and fragmentation barriers in full detail. Our models show that, consistent with current beliefs, the combination of radial drift and fragmentation is a strong limitation to growth of aggregates in disks. Typically, aggregates cannot grow to sizes larger than millimeters throughout the disk if threshold fragmentation velocities up to several $\mathrm{m} / \mathrm{s}$ are considered, though the precise maximum size is disk model dependent. This statement holds regardless of the amount of turbulence in the disk. For highly turbulent disks, it is the turbulence-induced relative velocities that cause the damage to aggregates, while for nearly non-turbulent disks it is the differential radial drift that limits the growth. Only if we set the fragmentation threshold velocity to the unlikely value of $30 \mathrm{~m} / \mathrm{s}$ or larger do we find that the fragmentation barrier can be broken and particles grow to larger sizes. Whether this high fragmentation threshold is realistic remains to be verified by high-speed laboratory collision experiments. It has been tentatively shown that high-speed impacts of a small projectile on a large target may result in growth (Wurm et al. 2005), but further study in this direction is imperative.

We have also investigated what happens when, for some reason, no fragmentation occurs. We then find that even in this reduced model, the particle radius never exceeded several centimeters at any time at any radius in the disk because of radial particle motion. However, we demonstrated that this radial drift barrier problem is very sensitive to slight changes in the initial dustto-gas ratio. If slighly higher initial dust-to-gas ratios than the canonical value of $1 \%$ are adopted in the simulations, then particles can grow to very large sizes in the inner parts of the disk.

We do not include non-linear feedback of the dust back onto the gas in our model. It has been recently shown by Johansen et al. (2007) that such feedback can lead to the rapid formation of gravitationally bound clumps of dust which subsequently form Ceres-size bodies. The "dust" particles, however, must be large (Stokes number near unity) before this scenario can take 
place. We find that for low-turbulent disks Stokes numbers larger than 0.1 can be reached, but we need further investigation if the amount of dust present in these large grains is sufficient to trigger such a gravitational collapse in locally overdense regions in the midplane of the disk.

In the full model, most of the solid material has drifted into the evaporation zone after $1 \mathrm{Myr}$ and the remnant disk contains less than $5 \%$ of the initial dust mass. However, there are reasons to believe that the strong radial drift of particles in such disks may be reduced by non-linear hydrodynamic effects. Tentative results from Johansen et al. (2006) find a reduction by a factor of 3 in MRI turbulence. Moreover, Brauer et al. (2007) show that observations of millimeter grains in the outer regions of protoplanetary disks indicate that the standard radial drift formulae are inconsistent with the observations. Hence, it may be important to investigate what happens to our model if radial drift velocities are reduced by some factor. This will be the topic of a future paper.

The dynamic calculations of Barge \& Sommeria (1995) and Klahr \& Henning (1997) also suggest that particle trapping in pressure maxima could be a solution also to the relative velocity fragmentation barrier. We intend, in future work, to study how the dust evolves if such pressure maxima are present.

By being able to model the dust evolution in a self-consistent way, our code may act as a basis upon wich further modeling of disks is done. For example, models of chemistry in disks are very dependent on the total surface area of dust grains per unit volume, in particular when grain surface chemistry is taken into account (Aikawa \& Herbst 1999; Semenov et al. 2006). Models of MRI turbulence in disks depend strongly on the abundance of free electrons. This abundance is also very dependent on the total surface area of dust (Ilgner \& Nelson 2006) and our model could - possibly with an ad-hoc reduction factor for the drift speed - provide new insight in this kind of modeling.

Our disk model involves a constant threshold fragmentation velocity which can be put into question. Laboratory experiments show that this threshold velocity is dependent on particle size (Blum \& Muench 1993; Paraskov et al. 2007) and we indeed work on that topic to investigate if more realistic aggregate collision models predict a different disk evolution (Brauer et al. in prep.).

Finally, current state-of-the-art models of disk structure and their spectra and images relies on ad-hoc prescriptions of the dust spatial and size distribution. Models of the kind described here will be linked to radiative transfer calculations to investigate the effect of grain evolution on disk structure in the near future.

Acknowledgements. We wish to thank Anders Johansen, Andras Zsom, Vernesa Smolcic, Dimitri Semenov and Konrad Tristram for useful discussions and comments. We also wish to thank the anonymous referee for useful criticism that helped us improve the paper.

\section{References}

Aikawa, Y., \& Herbst, E. 1999, A\&A, 351, 233

Andrews, S. M., \& Williams, J. P. 2007, ApJ, 659, 705

Apai, D., Pascucci, I., Sterzik, M. F., et al. 2004, A\&A, 426, L53

Apai, D., Pascucci, I., Bouwman, J., et al. 2005, Science, 310, 834

Apai, D., Luhman, K., \& Liu, M. 2007, ArXiv Astrophysics e-prints

Balbus, S. A., \& Hawley, J. F. 1991, ApJ, 376, 214

Barge, P., \& Sommeria, J. 1995, A\&A, 295, L1

Beckwith, S. V. W., Henning, T., \& Nakagawa, Y. 2000, Protostars and Planets IV, 533
Benz, W. 2000, Space Sci. Rev., 92, 279

Blum, J., \& Muench, M. 1993, Icarus, 106, 151

Blum, J., \& Wurm, G. 2000, Icarus, 143, 138

Blum, J., Wurm, G., Poppe, T., \& Heim, L.-O. 1998, Earth Moon and Planets, 80,285

Borkowski, K. J., \& Dwek, E. 1995, ApJ, 454, 254

Bouwman, J., Meeus, G., de Koter, A., et al. 2001, A\&A, 375, 950

Brandenburg, A., Nordlund, A., Stein, R. F., \& Torkelsson, U. 1995, ApJ, 446, 741

Brauer, F., Dullemond, C. P., Johansen, A., et al. 2007, A\&A, 469, 1169

Ciesla, F. J. 2007, ApJ, 654, L159

Cuzzi, J. N., \& Weidenschilling, S. J. 2006, Particle-Gas Dynamics and Primary Accretion in Meteorites and the Early Solar System II (Univ. Arizona Press), 353

Cuzzi, J. N., Dobrovolskis, A. R., \& Champney, J. M. 1993, Icarus, 106, 102

Cuzzi, J. N., Hogan, R. C., Paque, J. M., \& Dobrovolskis, A. R. 2001, ApJ, 546, 496

D’Alessio, P., Calvet, N., Hartmann, L., Franco-Hernández, R., \& Servín, H. 2006, ApJ, 638, 314

Davis, D. R., \& Ryan, E. V. 1990, Icarus, 83, 156

Desch, S. J., Connolly, Jr., H. C., \& Moser, D. E. 2002, in Lunar and Planetary Institute Conference Abstracts, Lunar and Planetary Institute Conference Abstracts, 33, 1768

Dohnanyi, J. W. 1969, J. Geophys. Res., 74, 2531

Dominik, C., Blum, J., Cuzzi, J. N., \& Wurm, G. 2007, in Protostars and Planets V (Univ. Arizona Press), 783

Dominik, C., \& Tielens, A. G. G. M. 1997, ApJ, 480, 647

Draine, B. T., \& Lee, H. M. 1984, ApJ, 285, 89

Dubrulle, B., Morfill, G., \& Sterzik, M. 1995, Icarus, 114, 237

Dullemond, C. P., \& Dominik, C. 2004, A\&A, 421, 1075

Dullemond, C. P., \& Dominik, C. 2005, A\&A, 434, 971

Hartmann, L., Calvet, N., Gullbring, E., \& D’Alessio, P. 1998, ApJ, 495, 385

Hawley, J. F., Gammie, C. F., \& Balbus, S. A. 1995, ApJ, 440, 742

Hayashi, C. 1981, in Fundamental Problems in the Theory of Stellar Evolution (D. Reidel Publishing Co.), IAU Symp., 93, 339

Ilgner, M., \& Nelson, R. P. 2006, A\&A, 445, 205

Johansen, A., Klahr, H., \& Henning, T. 2006, ApJ, 636, 1121

Johansen, A., Oishi, J. S., Low, M.-M. M., et al. 2007, Nature, 448, 1022

Johansen, A., \& Youdin, A. 2007, ApJ, 662, 627

Kempf, S., Pfalzner, S., \& Henning, T. K. 1999, Icarus, 141, 388

Kessler-Silacci, J. E., Dullemond, C. P., Augereau, J.-C., et al. 2007, ApJ, 659, 680

Kitamura, Y., Momose, M., Yokogawa, S., et al. 2002, ApJ, 581, 357

Klahr, H. H., \& Henning, T. 1997, Icarus, 128, 213

Klahr, H., \& Brandner, W. 2006, Planet Formation (Cambridge University Press)

Kornet, K., Stepinski, T. F., \& Różyczka, M. 2001, A\&A, 378, 180

Kovetz, A., \& Olund, B. 1969, J. Atmospher. Sci., 26, 1060

Lecar, M., Podolak, M., Sasselov, D., \& Chiang, E. 2006, ApJ, 640, 1115

Markiewicz, W. J., Mizuno, H., \& Voelk, H. J. 1991, A\&A, 242, 286

Mathis, J. S., Rumpl, W., \& Nordsieck, K. H. 1977, ApJ, 217, 425

Mizuno, H., Markiewicz, W. J., \& Voelk, H. J. 1988, A\&A, 195, 183

Morfill, G. E. 1988, Icarus, 75, 371

Nakagawa, Y., Nakazawa, K., \& Hayashi, C. 1981, Icarus, 45, 517

Nakagawa, Y., Sekiya, M., \& Hayashi, C. 1986, Icarus, 67, 375

Natta, A., Testi, L., Calvet, N., et al. 2007, in Protostars and Planets V (Univ. Arizona Press), 767

Nomura, H., \& Nakagawa, Y. 2006, ApJ, 640, 1099

Ormel, C. W., \& Cuzzi, J. N. 2007, A\&A, 466, 413

Ormel, C. W., Spaans, M., \& Tielens, A. G. G. M. 2007, A\&A, 461, 215

Ossenkopf, V. 1993, A\&A, 280, 617

Paraskov, G. B., Wurm, G., \& Krauss, O. 2007, Icarus, 191, 779

Poppe, T., Blum, J., \& Henning, T. 1999, Adv. Space Res., 23, 1197

Przygodda, F., van Boekel, R., Ầbrahàm, P., et al. 2003, A\&A, 412, L43

Reyes-Ruiz, M. 2007, MNRAS, 380, 311

Rodmann, J., Henning, T., Chandler, C. J., Mundy, L. G., \& Wilner, D. J. 2006, A\&A, 446, 211

Safronov, V. S. 1969, Evoliutsiia doplanetnogo oblaka

Sano, T., Inutsuka, S., Turner, N. J., \& Stone, J. M. 2004, Progr. Theor. Phys. Suppl., 155, 409

Schmitt, W., Henning, T., \& Mucha, R. 1997, A\&A, 325, 569

Schräpler, R., \& Henning, T. 2004, ApJ, 614, 960

Semenov, D., Wiebe, D., \& Henning, T. 2006, ApJ, 647, L57

Shakura, N. I., \& Sunyaev, R. A. 1973, A\&A, 24, 337

Sicilia-Aguilar, A., Hartmann, L. W., Watson, D., et al. 2007, ApJ, 659, 1637

Sirono, S.-I. 2004, Icarus, 167, 431

Smoluchowski, M. V. 1916, Z. Phys., 17, 557

Takeuchi, T., \& Lin, D. N. C. 2002, ApJ, 581, 1344 
Tanaka, H., Himeno, Y., \& Ida, S. 2005, ApJ, 625, 414

Tanaka, H., Inaba, S., \& Nakazawa, K. 1996, Icarus, 123, 450

Testi, L., Natta, A., Shepherd, D. S., \& Wilner, D. J. 2003, A\&A, 403, 323

van Boekel, R., Waters, L. B. F. M., Dominik, C., et al. 2003, A\&A, 400, L21

Völk, H. J., Morfill, G. E., Roeser, S., \& Jones, F. C. 1980, A\&A, 85, 316

Weidenschilling, S. J. 1977a, MNRAS, 180, 57

Weidenschilling, S. J. 1977b, Ap\&SS, 51, 153

Weidenschilling, S. J. 1980, Icarus, 44, 172

Weidenschilling, S. J. 1984, Icarus, 60, 553

Weidenschilling, S. J. 1988, Formation processes and time scales for meteorite parent bodies in Meteorites and the Early Solar System (Univ. Arizona Press), 348
Weidenschilling, S. J., \& Cuzzi, J. N. 1993, in Protostars and Planets III (Univ. Arizona Press), 1031

Whipple, F. L. 1972, in From Plasma to Planet (New York: Wiley Interscience Division), 211

Wilner, D. J., D’Alessio, P., Calvet, N., Claussen, M. J., \& Hartmann, L. 2005, ApJ, 626, L109

Wurm, G., Paraskov, G., \& Krauss, O. 2005, Icarus, 178, 253

Youdin, A. N. 2004, in Star Formation in the Interstellar Medium: In Honor of ed. D. Hollenbach, C. McKee, \& F. Shu (Francisco: Astronomical Society of the Pacific), ASP Conf. Ser., 323, 319

Youdin, A. N., \& Goodman, J. 2005, ApJ, 620, 459

Youdin, A. N., \& Lithwick, Y. 2007, ArXiv e-prints, 707 


\section{Appendix A: Coagulation algorithms}

\section{A.1. Podolak algorithm}

In this section we present an algorithm that was first used be Kovetz \& Olund (1969) in meteorological science. This algorithm conserves mass and particle number density. It captivates by its simplicity and it is comparatively easy to implement into a computer code.

Let us assume a mass grid $m_{i}$ and a given spatial number density $N_{i}$ of the particles with mass $m_{i}$. Two particles of mass $m_{i}$ and $m_{j}$ coagulate with a coagulation rate $Q_{i j}$, i.e. the number of coagulation events per time, which is given by

$Q_{i j}=N_{i} N_{j} K_{i j}$.

The quantity $K_{i j}$ denotes the coagulation kernel of the particles in the mass bins $i$ and $j$. It is given by the product of the collisional cross section $\sigma_{i j}$ between the particles and their relative velocity $v_{i j}$. High values of this kernel correspond to high collision rates which means that the particles are subject to a fast growth process. Low values of this quantity imply slow growth rates.

Now, an important issue appears if non-linear mass grids are considered. The resulting mass of the coagulation, i.e. the mass $m=m_{i}+m_{j}$, does not neccessarily match with any of the mass grid points. This means that in general no mass grid point $m_{\mathrm{s}}$ can be found which satisfies $m_{\mathrm{s}}=m_{i}+m_{j}$. Therefore, we have to divide the coagulating mass between the nearest mass grid points in some sensible way.

We assume that the nearest neighbours are given by $m_{m}<m<m_{n}$. With a linear ansatz we split the coagulation rate $Q_{i j}$ into a coagulation rate for the mass $m_{m}$ and a coagulation rate for the mass $m_{n}$,

$Q_{m}=\epsilon Q_{i j} \quad$ and $\quad Q_{n}=(1-\epsilon) Q_{i j}$

The number density is a conserved quantity in this algorithm since $Q_{m}+Q_{n}=Q_{i j}$. Much more important than the conservation of number density is the conservation of mass. We can enforce this fundamental conservation principle by setting

$Q_{m} m_{m}+Q_{n} m_{n}=Q_{i j}\left(m_{i}+m_{j}\right)$.

The last expression defines the value of $\epsilon$ which was a free parameter till now. Inserting Eqs. (A.2) into Eq. (A.3) we find that $\epsilon$ can be written as

$\epsilon=\frac{m_{n}-\left(m_{i}+m_{j}\right)}{m_{n}-m_{m}}$

One may translate these $\epsilon$ parameters for every coagulation process between particles of species $i$ and $j$ into certain coefficients $C_{i j k}$ so that the coagulation equation can be expressed in the form

$\dot{N}_{k}=\frac{1}{2} \sum_{i j} Q_{i j} C_{i j k}-\sum_{i} Q_{i k}$

The quantity $C$ is then given by

$C_{m i j k}= \begin{cases}\epsilon & \text { if } m_{k} \text { is the largest mass grid point }<m_{i}+m_{j} \\ 1-\epsilon & \text { if } m_{k} \text { is the smallest mass grid point }>m_{i}+m_{j} . \\ 0 & \text { otherwise }\end{cases}$

In general, more than $90 \%$ of the elements of the matrix $C$ are zero. Therefore, a lot of computer calculation time can be saved if only the non-zero elements in the last expression are summed up.

\section{A.2. Modified Podolak algorithm}

In simulations for protoplanetary disks, we are interested in particle growth from sub-micrometer in size, i.e. particles that are part of the interstellar medium, up to several hundred meters. This means that particle masses ranging from $10^{-12} \mathrm{~g}$ to $10^{12} \mathrm{~g}$ are considered, which corresponds to 24 orders of magnitude in mass.

Now, a major problem appears. Intrinsic computer variables, so-called double precision variables, have an accuracy only up to 14 digits. Since we are interested in particle growth by more than 20 orders of magnitude, the accuracy needed for coagulation simulations exceeds the accuracy provided by the computer. One solution could be the introduction of quadrupole precision computer variables but this would slow down the simulation speed significantly. This accuracy issue leads to certain problems for example the violation of mass conservation or simulation crashes. In order to perform the coagulation simulations anyway we have to analytically reformulate the Podolak algorithm at certain points of the numerical scheme.

For this purpose we first have to introduce a number $c_{e}$ in the following way. We consider the neighboring mass grid points $m_{k-1}$ and $m_{k}$. The number $c_{e}$ is then defined in a way that the inequality

$m_{k-1}+m_{i}<m_{k}$

holds for any $i$ which satisfies the condition $i \leq k-c_{e}$. In general, the value of $c_{e}$ is dependent on the index $k$. 
Now, we reformulate the algorithm. We formally separate the diagonal elements of Eq. (A.5) from the non-diagonal elements.

$\dot{N}_{k}=\frac{1}{2} \sum_{i=1}^{k} N_{i}^{2} C_{i i k} K_{i i}+\sum_{i=1}^{k} \sum_{j=1}^{i-1} N_{i} N_{j} C_{i j k} K_{i j}-\sum_{j=1}^{N} N_{j} N_{k} K_{j k}$.

In the next step, we consider the second and third summand of the last expression. In the second term we separate the case $i=k$ which leads to

$\sum_{i=1}^{k-1} \sum_{j=1}^{i-1} N_{i} N_{j} C_{i j k} K_{i j}+\sum_{j=1}^{k+1-c_{e}} N_{k} N_{j} C_{k j k} K_{k j}-\sum_{j}^{N} N_{j} N_{k} K_{j k}$

This can be rewritten as

$$
\begin{aligned}
& \sum_{i=1}^{k-1} \sum_{j=1}^{i-1} N_{i} N_{j} C_{i j k} K_{i j}+\sum_{j=1}^{k+1-c_{e}}\left(N_{k} N_{j} C_{k j k} K_{k j}-N_{j} N_{k} K_{j k}\right)-\sum_{j=k+2-c_{e}}^{N} N_{j} N_{k} K_{j k} \\
& =\sum_{i=1}^{k-1} \sum_{j=1}^{i-1} N_{i} N_{j} C_{i j k} K_{i j}-\sum_{j=1}^{k+1-c_{e}} N_{k} N_{j} K_{k j} \frac{m_{j}}{m_{k+1}-m_{k}}-\sum_{j=k+2-c_{e}}^{N} N_{j} N_{k} K_{j k} \\
& =\sum_{i=1}^{k-1} \sum_{j=1}^{i-1} N_{i} N_{j} C_{i j k} K_{i j}+\sum_{j=1}^{N} N_{k} N_{j} K_{k j} D_{j k},
\end{aligned}
$$

where the matrix $D$ is given by

$D_{j k}= \begin{cases}-\frac{m_{j}}{m_{k+1}-m_{k}} & \text { if } j \leq k+1-c_{e} \text { and } \\ -1 & \text { if } j>k+1-c_{e} .\end{cases}$

The new coagulation equation now reads

$\dot{N}_{k}=\frac{1}{2} \sum_{i=1}^{k} N_{i}^{2} C_{i i k} K_{i i}+\sum_{i=1}^{k-1} \sum_{j=1}^{i-1} N_{i} N_{j} C_{i j k} K_{i j}+\sum_{j=1}^{N} N_{k} N_{j} K_{k j} D_{j k}$.

This was one part of rewriting the algorithm. For the other part we regard the second term of the last expression, especially the term $i=k-1$. We can rewrite this term as follows,

$$
\begin{aligned}
\sum_{j=1}^{k-2} N_{k-1} N_{j} C_{k-1, j, k} K_{k-1, j} & =\sum_{j=1}^{k-c_{e}} N_{k-1} N_{j} C_{k-1, j, k} K_{k-1, j}+\sum_{k-c_{e}+1}^{k-2} N_{k-1} N_{j} C_{k-1, j, k} K_{k-1, j} \\
& =\sum_{j=1}^{k-c_{e}} N_{k-1} N_{j} \frac{m_{j}}{m_{k}-m_{k-1}} K_{k-1, j}+\sum_{k-c_{e}+1}^{k-2} N_{k-1} N_{j} C_{k-1, j, k} K_{k-1, j} \\
& =\sum_{j=1}^{k-2} N_{k-1} N_{j} K_{k-1, j} E_{j k} \\
& =\sum_{i=1}^{N} \sum_{j=1}^{N} N_{i} N_{j} K_{i j} E_{j, i+1} \theta\left(k-j-\frac{3}{2}\right) \delta_{i, k-1} .
\end{aligned}
$$

In this equation the matrix $E$ is given by

$E_{j k}= \begin{cases}\frac{m_{j}}{m_{m}-m_{k-1}} & \text { if } j \leq k-c_{e} \text { and } \\ {\left[1-\frac{m_{j}+m_{k-1}-m_{k}}{m_{k+1}-m_{k}}\right] \theta\left(m_{k+1}-m_{j}-m_{k-1}\right)} & \text { if } j>k-c_{e} .\end{cases}$

With these two reformulations the coagulation equation can be written in the form

$\dot{N}_{k}=\sum_{i j} N_{i} N_{j} K_{i j} M_{i j}$,

where the final coagulation matrix $M$ is given by

$M_{i j}=\frac{1}{2} \delta_{i j} C_{i j k}+C_{i j k} \Theta\left(k-i-\frac{3}{2}\right) \Theta\left(i-j-\frac{1}{2}\right)+\delta_{i k} D_{j i}+\delta_{i, k-1} E_{j, i+1} \Theta\left(k-j-\frac{3}{2}\right)$.

In this expression $\Theta(x)$ denotes the Heaviside distribution, which is zero for $x<0$ and unity for $x>1$. 


\section{Appendix B: Vertical integration}

Coagulation and fragmentation are local processes. This means that the equations described in the last section have to be solved at every point in space. The more space grid points are considered the more time-consuming the computer simulations become. However, under certain conditions the situation simplifies. In the following we will describe a scheme that can save a remarkable amount of computational time.

We consider the coagulation (fragmentation) equation at a certain space point $z_{p}$

$\dot{N}_{k}\left(z_{p}\right)=\sum_{i j} G_{i j k}\left(z_{p}\right) N_{i}\left(z_{p}\right) N_{j}\left(z_{p}\right)$.

Since we are interested in particle growth in protostellar disks we can adapt the number densities to this special problem. We assume that at any given time the vertical particle distribution of any given particle size is given by a settling-mixing equilibrium distribution (cf. Eq. (15)). This leads to a density $N_{i}$ of a particle of size $a_{i}$ which depends on the height above the midplane $z$ as

$N_{i}(z)=\frac{\omega_{i}}{\sqrt{2 \pi} h_{i}} \exp \left[-\frac{1}{2}\left(\frac{z}{h_{i}}\right)^{2}\right]$.

In this expression the variable $h_{i}$ denotes the dust scale height of the particles with mass $m_{i}$. The quantity $\omega_{i}$ is the surface number density of the particles with that certain mass. Inserting Eqs. (B.2) into (B.1) and integrating over height above the midplane $z$ yields

$\dot{\omega}_{k}=\sum_{i j} \omega_{i} \omega_{j} \sum_{p} \frac{G_{i j k}\left(z_{p}\right)}{2 \pi h_{i} h_{j}} \exp \left[-\frac{1}{2}\left(\frac{z_{p}}{h_{i}}\right)^{2}\right] \exp \left[-\frac{1}{2}\left(\frac{z_{p}}{h_{j}}\right)^{2}\right] \Delta z_{p}$.

If we define

$\tilde{G}_{i j k}=\sum_{p} \frac{G_{i j k}\left(z_{p}\right)}{2 \pi h_{i} h_{j}} \exp \left[-\frac{1}{2}\left(\frac{z_{p}}{h_{i}}\right)^{2}\right] \exp \left[-\frac{1}{2}\left(\frac{z_{p}}{h_{j}}\right)^{2}\right] \Delta z_{p}$,

the integrated coagulation equation can be written as

$\dot{\omega}_{k}=\sum_{i j} \omega_{i} \omega_{j} \tilde{G}_{i j k}$

In this way we have integrated the $z$-dimension out without a single approximation only with the assumption that the vertical redistribution goes faster than the coagulation/fragmentation. This reformulation of the coagulation equation has an obvious advantage, namely instead of solving the coagulation equation at every point in $z$ the last expression enables us to solve the equation for every height above the midplane at the same time. If we assume a vertical grid with 60 grid points the vertical integration speeds up the computer simulation routine by a factor of 60 .

\section{Appendix C: Implicit differencing}

If fragmentation is included in the simulations then the limiting time step for the coagulation/fragmentation process tends to be small. Fragmentation leads to a permanent amount of small particles. Small particles, however, are associated with short time scales. Taking these short time scales into account, the time step of the numerical simulation can not be chosen to be very large. This argumentation only holds for explicit numerical solvers. For this reason we have implemented an implicit solver for the coagulation/fragmentation equation which we will describe in the following.

The coagulation/fragmentation equation can be written in the form

$\bar{f}=\bar{F}(\bar{f})$,

where $\bar{f}$ denotes the particle distribution vector on the mass grid and the function $\bar{F}$ describes the time evolution. In one time step $\Delta t$ at a certain time $t$, we now want to calculate the new particle distribution $\bar{f}_{\mathrm{n}}=\bar{f}(t+\Delta t)$ from the old distribution $\bar{f}_{\mathrm{o}}=\bar{f}(t)$. Therefore we rewrite Eq. (C.1) as

$\bar{\epsilon}=\Delta t \bar{F}\left(\bar{f}_{i}\right)$,

where $\bar{\epsilon}=\bar{f}_{\mathrm{n}}-\bar{f}_{\mathrm{o}}$ and $\bar{f}_{\mathrm{i}}=\xi \bar{f}_{\mathrm{o}}+(1-\xi) \bar{f}_{\mathrm{n}}$. The time evolution of the function $\bar{f}$ with $\xi=1$ is called explicit, while the time evolution with $\xi=0$ is usually called implicit. Choosing $\xi=0$ in our case, we can perform a Taylor expansion of the right-hand side of Eq. (C.2) which leads to

$\bar{\epsilon}=\Delta t \bar{F}\left(\bar{f}_{\mathrm{o}}\right)+\Delta t \tilde{J} \bar{\epsilon}$.

The Matrix $\tilde{J}$ denotes the Jacobi matrix which is definded as $\tilde{J}_{i j}=\partial F_{i} / \partial f_{j}$. Solving Eq. (C.3) for $\bar{\epsilon}$ leads to

$\bar{\epsilon}=[1-\Delta t \tilde{J}]^{-1} \Delta t \bar{F}\left(\bar{f}_{\mathrm{o}}\right)$.

Hence, the evolution of the implicit time step reduces to a matrix inversion which can be done easily. 
F. Brauer et al.: Coagulation and fragmentation of grains. Online Material $p 4$

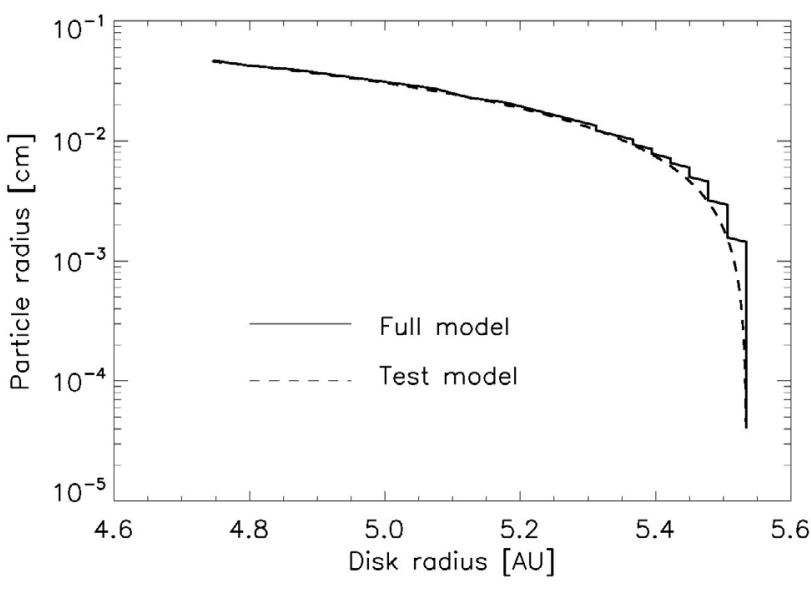

Fig. D.1. Test for the radial drift routine. Coagulation is neglected in this simulation. The lines denote the particle position in the disk after $10^{3} \mathrm{yrs}$ of radial drift from the initial radial position $r_{0}=5.53 \mathrm{AU}$ for different dust particle sizes.

\section{Appendix D: Tests}

This part of the appendix considers a comparison between the model described in Sect. 2 and a model which involves the following approximation. The particle distributions which can be seen for example in Fig. 6 are fairly narrow peaks. Hence, it is suggestive to approximate the particle distribution by a monodisperse distribution, i.e. a distribution with only one single particle size. In this case the coagulation equation simplifies enormously. We will compare these two different models by considering coagulation due to Brownian motion and turbulent coagulation. Also the routine for radial motion will be checked against this simplified model.

\section{D.1. Simplified model and numerical setup}

We assume that a certain amount of equally-sized dust particles with radius $a$ is located at a single radius $r$ in the disk. The surface density of these particle is given by $\Sigma_{\mathrm{d}}$. The dust particles are vertically distributed according to Eq. (15). For the test cases we ignore the radial motion of the gas and the radial turbulent diffusion of the dust so that the radial motion of the dust is given by Eq. (17). The assumption of a monodisperse distribution leads to a coagulation equation given by Eq. (34) (Kornet et al. 2001). In the test cases we will use a surface density of the dust $\Sigma_{\mathrm{d}}=5.26 \times 10^{-2} \mathrm{~g} / \mathrm{cm}^{2}$ and an initial location in the disk given by $r_{0}=5.53 \mathrm{AU}$. Every other parameter was already mentioned in Sect. 2.

\section{D.2. Radial drift}

First, we check the radial drift routine. Any coagulation is neglected. With the values mentioned above we let the dust particles drift for $10^{3} \mathrm{yrs}$ and plot their radial position in the disk for different particle sizes. The results of this simulation are shown in Fig. D.1. In this figure the solid line correponds to the dominant particle size of the dust distribution in the full model. The dashed line denotes the result of the monodisperse model. Any discontinuous effects are due to the radial grid.

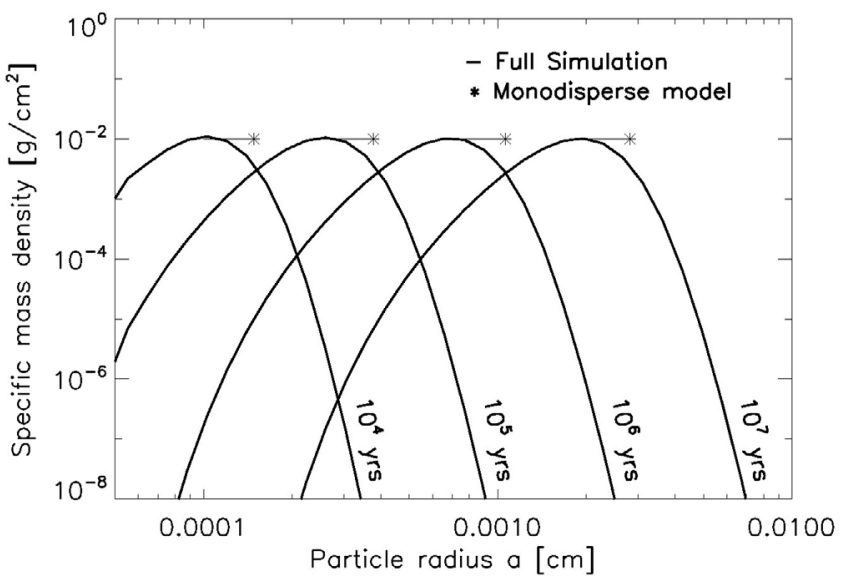

Fig. D.2. Test for the coagulation routine. This plot shows the particle distribution in the full model and the monodisperse model for coagulation due to Brownian motion at 4 different times. The stars " $\star$ " denote the particle size in the monodisperse model. The largest discrepancy in particle size between the two models is a factor of 1.6 in particle size $a$.

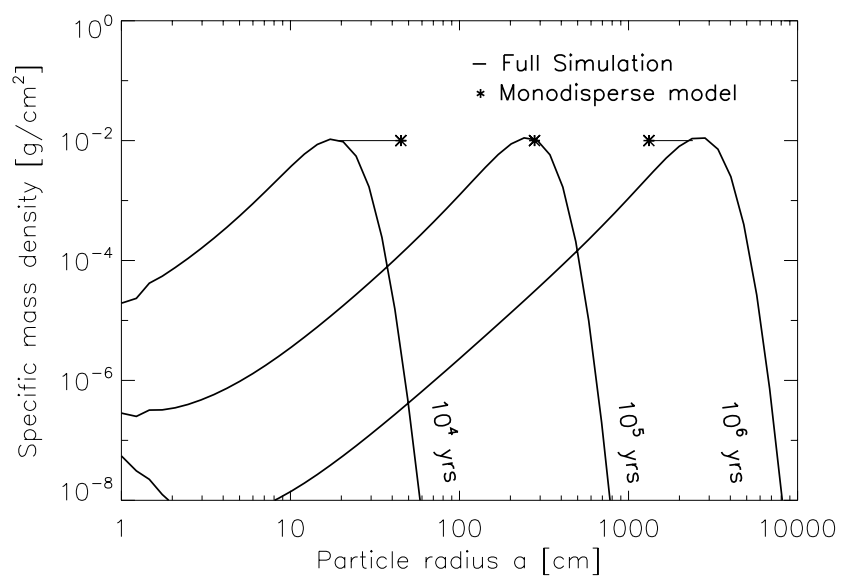

Fig. D.3. Test for the coagulation routine. This plot shows the particle distribution in the full model and the monodisperse model for coagulation due to Brownian motion and turbulence in the disk at 3 different times. The stars " $\star$ " denote the particle size in the monodisperse model. The largest discrepancy in particle size between the results that are shown in this figure is a factor of $\sim 2.7$.

\section{D.3. Coagulation}

Now, we consider the coagulation of the dust while the radial motion of the dust is neglected. We investigate the dust particle coagulation at $r_{0}=5.53 \mathrm{AU}$ in the disk and we first focus on coagulation due to Brownian motion. The resul " $\star$ " denote the particle size in the monodisperse model. The largest discrepancy in particle size between the two models is a factor of $\sim 1.6$ after $10^{7}$ yrs.

The results of the same simulation but now with particle growth due to turbulent coagulation included are shown in Fig. D.3. The largest discrepancy in this case in particle size between the two models is a factor of $\sim 2.7$ in radius for $t=10^{4} \mathrm{yrs}$.

However, if the Stokes number of the dust particles is smaller than unity, then the particle size of both models after a certain time can differ in more than one order of magnitude. This is due to the following reason. If the Stokes number is smaller than unity then the relative turbulent velocity of the dust follows

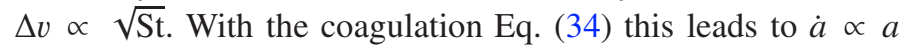
and its solution $a \propto \exp (t)$. In the particular case of turbulent 
coagulation and $\mathrm{St}<1$, the particle size as a function of time is not given by a polynomial expression, i.e. an expression of the form $a \propto t^{\gamma}$, but an exponential function. If a time evolution follows a polynomial law then two different but similar initial conditions will lead to different but similar particle sizes after any time. Let us consider the time evolution

$a(t)=\left[c t+a_{0}^{1 / \gamma}\right]^{\gamma}$.

First, the initial particle size $a_{0}$ gets unimportant for large $t$. Second, two different $c$-parameters, i.e. $c_{1}$ and $c_{2}$, will lead to particle sizes which will differ by a factor of $\left(c_{1} / c_{2}\right)^{\gamma}$. This factor is constant and does not increase.

On the other hand, if the particles grow exponentially,

$a(t)=a_{0} \exp (c t)$

then the initial particle size $a_{0}$ will always play a role. Moreover, two different c-values, i.e. $c_{1}$ and $c_{2}$, will lead to particle sizes which will differ by a factor of $\exp \left(c_{1} t-c_{2} t\right)$. This factor increases in time. For example, in the case of turbulent coagulation (cf. Eq. (39)) the parameter $c$ is given by $\epsilon_{0} \Omega_{\mathrm{k}}$. An initial dust-to-gas ratio of $1 \%$ leads to $a=a_{0} e \approx 2.72 a_{0}$ after 100 orbits. An initial dust-to-gas ratio of $2 \%$ after 200 orbits already implies $a=a_{0} e^{4} \approx 54.6 a_{0}$ which is a factor of 20 larger. In the case of turbulent coagulation and St smaller than unity, small changes in the intial conditions lead to large differences in the growth behaviour. Hence, a change from a monodisperse model to a model with a whole particle dispersion presumably leads to similar effects.

\section{Appendix E: Collision rates}

In this part of the Appendix, we investigate the equilibrium particle distribution between coagulation and fragmentation after $10^{3}$ yrs of disk evolution at $1 \mathrm{AU}$ in the disk. In this calculation the fragmentation velocity is $v_{\mathrm{f}}=10^{3} \mathrm{~cm} / \mathrm{s}$ and the fragmentation parameter $\xi$ is 1.83 . We adopt a disk mass of $10^{-2} M_{\star}$, a turbulent $\alpha$-value of $10^{-3}$ and an initial dust-to-gas ratio of $10^{-2}$. The cratering parameter $\chi=0.5$ and we adopt $\psi=2$. We focus on the collision rates between particles of different sizes and how important these collisions are for particle growth.

We define the collision rates $R\left(r_{1}, r_{2}\right)$ as the number of collisions per second between particles of radius $r_{1}$ and $r_{2}$ in a vertical column with a cross section of $1 \mathrm{~cm}^{2}$. These collision rates are shown in Fig. E.1. The collision rates for particles of equal size decrease dramatically with increasing particle radius. The collision rates of micrometer-sized particles and the collision rates of mm-sized particles differ by more than 10 orders of magnitude. While the collision rate for $1 \mu \mathrm{m}$ particles is $\sim 10^{4 \ldots 5} \mathrm{~cm}^{-2} \mathrm{yrs}^{-1}$, the collision rate for $10 \mu \mathrm{m}$ particles is already more than two orders of magnitude lower. The smallest particles have the highest collision rates. Since the coagulation probability for $\mu \mathrm{m}$ particles is rather high, this leads to relatively short coagulation time scales for small particles. The collision rate is $1 \mathrm{~cm}^{-2} \mathrm{yrs}^{-1}$ for $10^{2} \mu \mathrm{m}$ particles. For particles, which are one order of magnitude in radius larger, this rate has already dropped to $10^{-6} \mathrm{~cm}^{-2} \mathrm{yrs}^{-1}$. However, the behaviour of the collision rates between non-equal sizes particles is different. For example, the collision rate between $\sim \mu$ m-sized dust particles and larger particles does hardly change over a wide range.

The collision rates, which are shown in Fig. E.1, do not provide information about the importance of collisions for particle growth. Not only the number of collisions per time is important for particle coagulation, but also the mass of the particles itself.

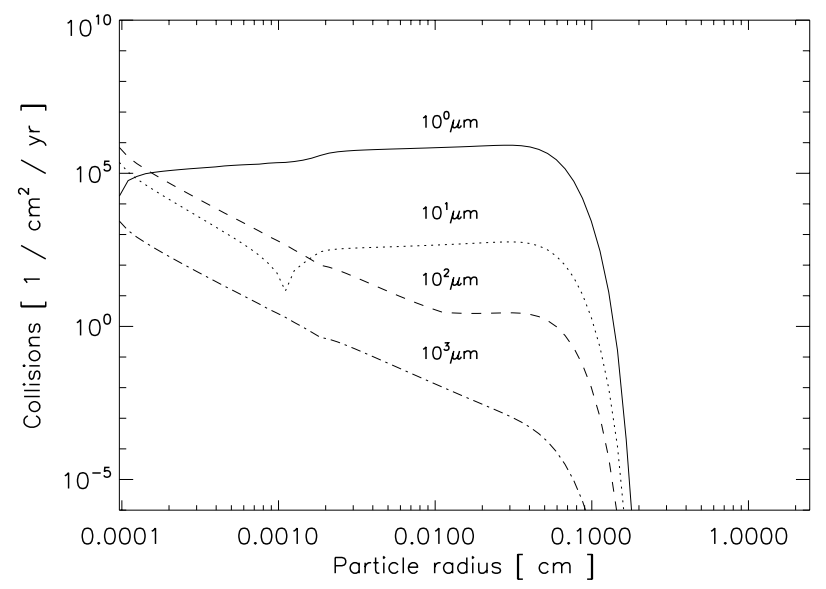

Fig. E.1. This plot shows the vertically integrated number of collisions per time at $1 \mathrm{AU}$ in the disk as discussed in Sect. E. This calculation is based on the particle distribution after $10^{3}$ yrs of disk evolution shown in Fig. 13. In this simulation we adopted a disk mass of $10^{-2} M_{\star}$ and a turbulent $\alpha$ parameter of $10^{-3}$.

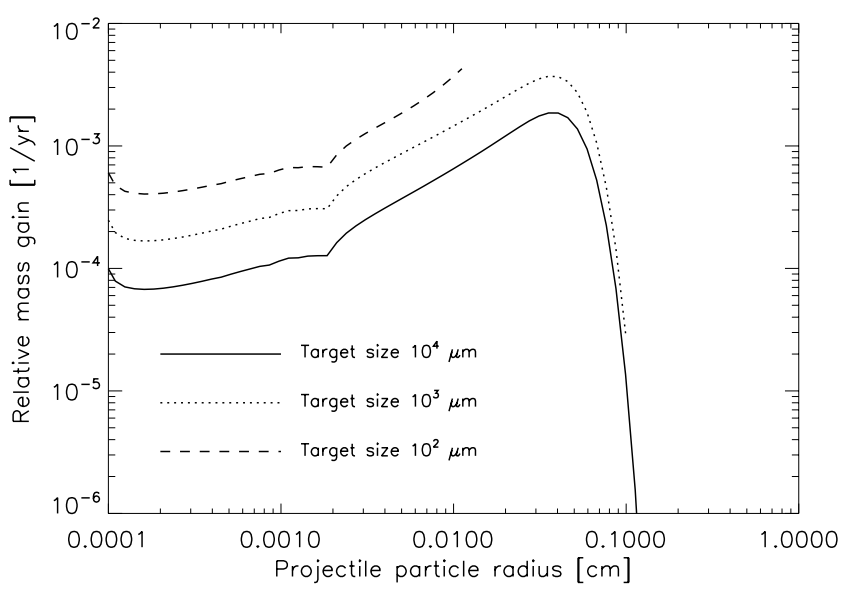

Fig. E.2. This plot shows the relative mass gain per year at $1 \mathrm{AU}$ in the disk. This calculation is based on the particle distribution after $10^{3} \mathrm{yrs}$ of disk evolution. The disk mass is $10^{-2} M_{\star}$ and the turbulent $\alpha$ parameter is $10^{-3}$.

For this reason, we calculate the relative mass gain of a single particle with radius $r_{1}$. If the number of all particles of radius $r_{1}$ in a vertical column is given by $N_{1}$, then in average every single particle of size $r_{1}$ collides with $R\left(r_{1}, r_{2}\right) / N_{1}$ particles of size $r_{2}$ per second. We assume that the sticking probability is unity. This means that the mass, which the particle of radius $r_{1}$ sweeps up in time, is given by $R\left(r_{1}, r_{2}\right) m_{2} / N_{1}$. Its relative mass gain rate is then given by

$\Xi_{r_{1}}\left(r_{2}\right)=\frac{R\left(r_{1}, r_{2}\right)}{N_{1}} \frac{m_{2}}{m_{1}}$

This quantity is shown in Fig. E.2. Since larger particles sweep up smaller particles the mass gain rates $\Xi$ are not shown for particle sizes $r_{2}>r_{1}$.

We find that the collisions which are most important for particle growth are collisions between equal-sized particles. This statement holds for particles smaller than $\sim 0.5 \mathrm{~mm}$ in size. If the particle with radius $r_{1}$ is larger than this value then collisions with $r_{2} \sim 0.5 \mathrm{~mm}$ sized particles are most important for the growth of the dust. 
F. Brauer et al.: Coagulation and fragmentation of grains, Online Material p 6

Table E.1. Important variables used in the course of this paper.

\begin{tabular}{|c|c|}
\hline Variable & Explanation \\
\hline$a$ & radius of the particle \\
\hline$m$ & mass of the particle \\
\hline$\rho_{\mathrm{s}}$ & solid material density of the particle \\
\hline$r$ & distance to the central star from a point in the midplane \\
\hline$z$ & height above the midplane \\
\hline $\mathrm{T}$ & temperature \\
\hline$c_{\mathrm{s}}$ & isothermal soundspeed \\
\hline$\Omega_{\mathrm{k}}, V_{\mathrm{k}}$ & Kepler frequency, Kepler velocity \\
\hline$H=c_{\mathrm{s}} / \Omega_{\mathrm{k}}$ & gas scale height \\
\hline$h$ & dust scale height \\
\hline$\Sigma_{\mathrm{g}}, \Sigma_{\mathrm{d}}$ & surface density of the gas and the dust \\
\hline$\rho_{\mathrm{g}}, \rho_{\mathrm{d}}$ & gas and dust density \\
\hline$\epsilon_{0}, \epsilon$ & initial and current dust-to-gas ratio \\
\hline$r_{\text {in }}$ & inner radius of the disk \\
\hline$r_{\text {out }}$ & outer radius of the disk \\
\hline$M_{\star}$ & mass of the central star \\
\hline$M_{\text {disk }}$ & mass of the disk \\
\hline$v_{n}$ & maximum radial drift velocity \\
\hline$\alpha, q$ & turbulence parameters \\
\hline & Stokes number of the particle \\
\hline$D_{\mathrm{g}}, D_{\mathrm{d}}$ & diffusion coefficients of gas and dust \\
\hline$v_{\text {dust }}$ & radial dust drift velocity due to gas drag \\
\hline$v_{\text {gas }}$ & radial gas accretion velocity \\
\hline$v_{\text {dust }}^{\text {tot }}$ & total radial dust velocity \\
\hline$\xi$ & slope of the particle distribution after fragmentation \\
\hline$x$ & relative amout of mass removed from the target particle by cratering \\
\hline$v_{\mathrm{f}}$ & threshold fragmentation velocity \\
\hline
\end{tabular}

